\title{
Angular Momentum Transport in Stellar Interiors
}

\section{Conny Aerts, ${ }^{1,2}$ Stéphane Mathis,,${ }^{3,4}$ and Tamara M. Rogers ${ }^{5,6}$}

\footnotetext{
1 Institute of Astronomy, KU Leuven, Celestijnenlaan 200D, B-3001 Leuven, Belgium; email: Conny.Aerts@kuleuven.be 2 Department of Astrophysics, IMAPP, Radboud University Nijmegen, P.O. Box 9010, 6500 GL Nijmegen, The Netherlands

${ }^{3}$ Laboratoire AIM Paris-Saclay, CEA/DRF - CNRS - Universit Paris Diderot, IRFU/DAp Centre de Saclay, F-91191 Gif-sur-Yvette, France; Stephane.Mathis@cea.fr

${ }^{4}$ LESIA, Observatoire de Paris, PSL Research University, CNRS, Sorbonne Universités, UPMC Université Paris 06, Université Paris Diderot, Sorbonne Paris Cité, 5 place Jules Janssen, F-92195 Meudon, France

${ }^{5}$ Department of Mathematics, Statistics and Physics, Newcastle University, Newcastle upon Tyne, UK; Tamara.Rogers@newcastle.ac.uk

${ }^{6}$ Planetary Science Institute, Tucson, AZ 85721, USA
}

Xxxx. Xxx. Xxx. Xxx. 2019. 57:1-45

https://doi.org/10.1146/((please add article doi))

Copyright (C) 2019 by Annual Reviews. All rights reserved

\section{Keywords}

waves, asteroseismology, stars: oscillations (including pulsations), stars: interiors, stars: rotation, stars: evolution

\section{Abstract}

Stars lose a significant amount of angular momentum between birth and death, implying that efficient processes transporting it from the core to the surface are active. Space asteroseismology delivered the interior rotation rates of more than a thousand low- and intermediatemass stars, revealing that: 1) single stars rotate nearly uniformly during the core hydrogen and core helium burning phases; 2) stellar cores spin up to a factor 10 faster than the envelope during the red giant phase; 3 ) the angular momentum of the helium-burning core of stars is in agreement with the angular momentum of white dwarfs. Observations reveal a strong decrease of core angular momentum when stars have a convective core. Current theory of angular momentum transport fails to explain this. We propose improving the theory with a data-driven approach, whereby angular momentum prescriptions derived from multidimensional (magneto)hydrodynamical simulations and theoretical considerations are continously tested against modern observations. The TESS and PLATO space missions have the potential to derive the interior rotation of large samples of stars, including high-mass and metalpoor stars in binaries and clusters. This will provide the powerful observational constraints needed to improve theory and simulations. 


\section{Contents}

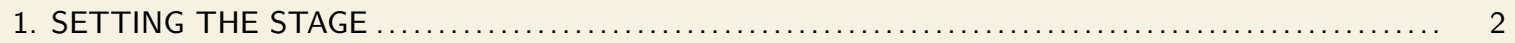

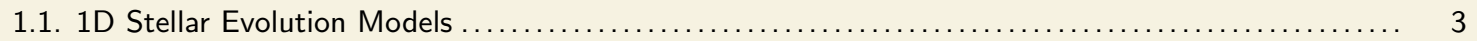

1.2. Classical Observational Constraints for Stellar Interiors $\ldots \ldots \ldots \ldots \ldots \ldots \ldots \ldots \ldots \ldots \ldots \ldots \ldots \ldots \ldots$

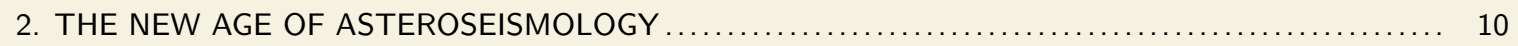

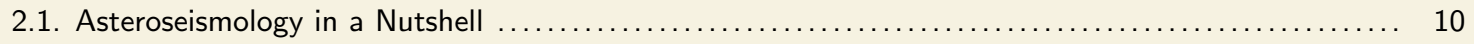

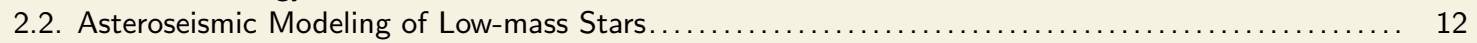

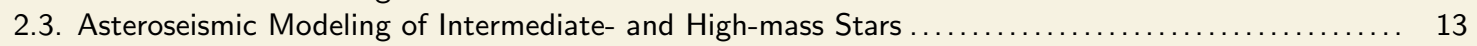

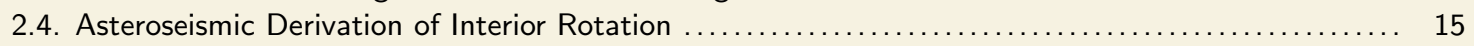

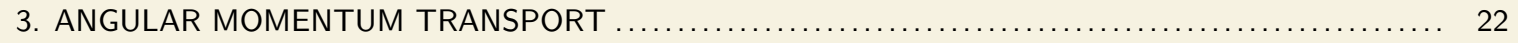

3.1. Hydrodynamical Meridional Circulation: from Eddington Sweet to Zahn's Model ............... 22

3.2. Hydrodynamical Instabilities and Convective Penetration/Overshoot $\ldots \ldots \ldots \ldots \ldots \ldots \ldots \ldots \ldots \ldots 23$

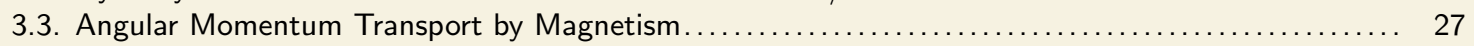

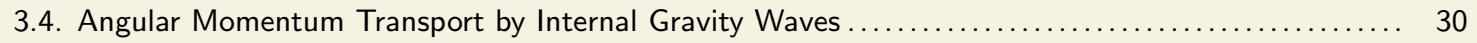

4. FUTURE OUTLOOK TO IMPROVE STELLAR MODELING $\ldots \ldots \ldots \ldots \ldots \ldots \ldots \ldots \ldots \ldots \ldots \ldots \ldots$

Low-mass star: a star that, at the onset of core hydrogen burning, has a radiative core or is fully convective $\left(M \lesssim 1.3 \mathrm{M}_{\odot}\right)$ and will end its life as a white dwarf

Intermediate-mass star: a

star that has a convective core at the onset of core hydrogen burning and that will end its life as a white dwarf $\left(1.3 \lesssim M \lesssim 8 \mathrm{M}_{\odot}\right)$

High-mass star: a star born with a large convective core and with sufficient mass $\left(M \gtrsim 8 \mathrm{M}_{\odot}\right)$ to undergo core collapse at the end of its life to form a neutron star or a black hole

\section{SETTING THE STAGE}

Stars are radiating rotating gaseous spheres whose interior properties meet the laws of gravity and hydrodynamics. Understanding the properties of stellar interiors up to present-day observational precision requires a complex interplay between atomic and nuclear physics, (quantum-)mechanics and thermodynamics, with intense interactions between radiation and matter. Moreover, the dynamics in stellar interiors and the life cycles of stars happen on length and time scales that span so many orders of magnitude that we remain very far from full 3D stellar evolution computations, even with the most powerful computers now or in the foreseeable future. We must therefore resort to $1 \mathrm{D}$ approximations when computing stellar evolution models.

Besides being fascinating in their own right, theoretical stellar structure and evolution models are basic ingredients for many areas of astrophysics. Indeed, stars are the building blocks of galaxies, clusters, associations, binaries, and exoplanetary systems. Therefore, these studies, in addition to stellar evolution theory, rely heavily on stellar models (and their accuracy). These models are constantly subjected to new observational constraints, as more, and particularly more precise, data become available. Such has happened with high-precision space photometry the past decade, with pleasant confirmation of how well some aspects of stellar models represent reality, but with unanticipated surprises revealing how poor the models are on other aspects.

In this review, we focus on one pertinent aspect of stellar models that turns out to be in need of major improvement: the angular momentum transport that occurs inside a star during its evolution. We treat this topic from three complementary aspects: modern observations, state-of-the art theory and multi-D numerical simulations. Each of these aspects is detailed below, with brief historical reminders. The historical path for these three aspects is quite different in duration and maturity, with theory going back almost a century, hydrodynamical simulations in 2D or 3D having started only when computational power allowed it since some 30 years, and direct observations of stellar interiors for stars other than the Sun and white dwarfs reaching sufficient precision only about a decade ago, when space asteroseismology came into practice. 
When it comes to predicting the type of remnant at the end of stellar life - a white dwarf for low- or intermediate-mass stars born with a mass $M \lesssim 8 \mathrm{M}_{\odot}$ and a neutron star or black hole for high-mass stars born with a mass $M \gtrsim 8 \mathrm{M}_{\odot}$ - the theory of stellar evolution is quite well established (e.g., Maeder 2009; Kippenhahn, Weigert \& Weiss 2012, for modern monographs). On the other hand, the theory of stellar interiors relies on physical concepts that are still subject to considerable uncertainties. Indeed, many of the (intrinsically multidimensional) phenomena connected with rotation, magnetism, mixing of chemical elements, and angular momentum transport inside stars cannot be deduced from first principles. Such physical ingredients therefore include one or more free parameters and these remained essentially uncalibrated prior to the asteroseismology era, because observational measurements were mostly limited to constraints coming from the stellar photosphere.

Long-standing theoretical concepts taken for granted for stellar structure computations, such as local conservation of angular momentum, rotational mixing, and dynamical instabilities (see the extensive monographs by, e.g., Hansen, Kawaler \& Trimble 2004; Maeder 2009) turn out to have unanticipated inaccuracies in terms of the transport of chemical elements and of angular momentum induced by them in the deep stellar interior. Theory and observations differ by two orders of magnitude with regard to core-to-envelope rotation rates in low-mass evolved stars, while the level of chemical mixing in young intermediatemass stars connected with instabilities in their radiative layers adjacent to the core are orders of magnitude lower than anticipated. These limitations of the models will be further outlined below. Many of these difficulties were only recently uncovered thanks to the probing capacity of stellar oscillations, offering a direct view of deep stellar interiors.

This review first offers a concise reminder of "classical" observational constraints from spectroscopy, interferometry, and astrometry of single, binary, and cluster stars used to calibrate stellar interiors, after a brief discussion of the differences between 1D stellar evolution models with and without rotation. Such classical diagnostics at best have relative precisions of order a few percent, but will remain an important observational method to evaluate various types of stellar models for stars and stellar systems that do not reveal oscillations suitable for asteroseismology. Next, we highlight the recent asteroseismic input from stars that offer the opportunity to calibrate stellar models, with specific emphasis on internal rotation. Finally, we place the new observational diagnostics into context, by providing a historical overview and the current status of the theory of transport processes in stars, detailing angular momentum transport in particular. We highlight the gain from a fully integrated approach combining observations, theory, and multi-D simultations into a global picture. Such a comprehensive approach requires the application and integration of a multitude of techniques and methods based on observations and theory. An encompassing visualisation is offered in Figure 1, where each of the keywords used to construct it will be discussed below. It represents graphically the conclusion of our review on the way forward towards a better understanding of angular momentum transport in stars.

\subsection{D Stellar Evolution Models}

As will be outlined in Section 2, we now have asteroseismic probes of the properties of stellar interiors. Such diagnostics give direct access to the physics at different depths inside the star, in particular the regions near the stellar core. Stellar models relying on physical descriptions that were taken for granted for decades, can now finally be calibrated asteroseismically. From this, it has been found that aspects of "well established" theory do not

J: a ngular momentum of a star, defined as $M \cdot \Omega \cdot R^{2}$ and expressed in the SI units $\mathrm{kg} \mathrm{m}^{2} \mathrm{~s}^{-1}$ 
Main sequence: $\mathrm{t}$ he phase in a star's evolution when it is burning hydrogen in its core

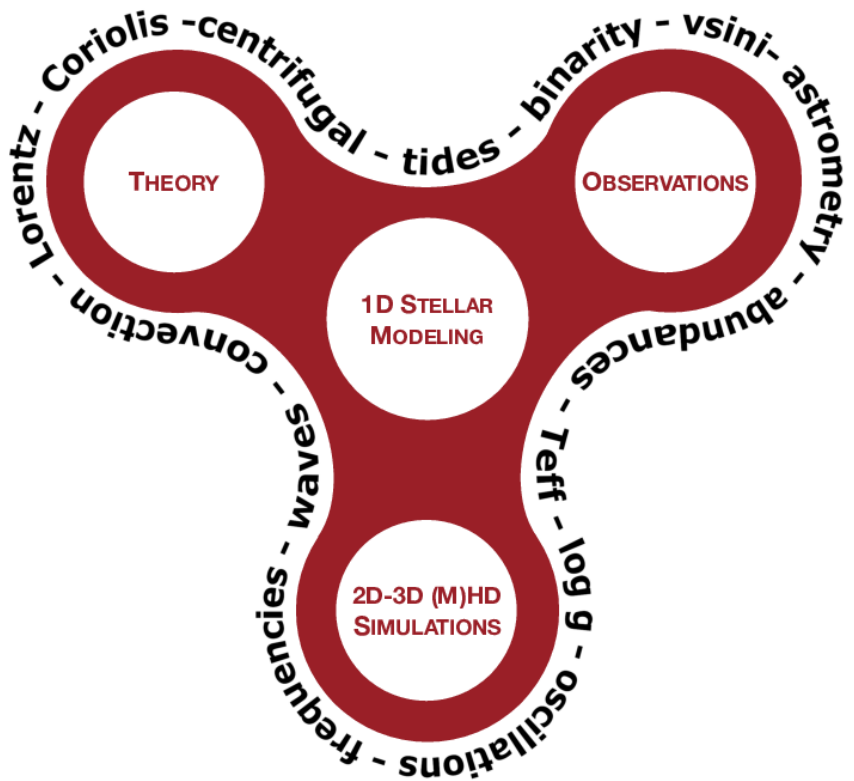

Figure 1

Synergies and complementarities between theory, various types of observations, and multi-D simulations. Bridging all those aspects will lead to an inclusive methodology for 1D stellar modeling and a better understanding of stellar interiors, including angular momentum transport.

meet the asteroseismic requirements, particulary in the case of rotation and angular momentum transport. In order to offer a path towards improved theory, we first remind the reader of some basic ingredients of stellar structure theory.

Stellar models are obtained by solving a set of differential equations based on the laws of physics, accompanied by proper boundary conditions for the center and surface of the star. Solving these equations requires that choices for the microscopic properties of stellar matter are made, in particular the equation of state of the gas, nuclear reactions, the interaction of radiation and matter, etc. Despite improved computational power the past decades, stellar models necessarily remain a simplified version of reality, because it is not possible to compute complete 3D models across stellar evolution. Pragmatic choices in the approximations adopted to solve the stellar structure equations are thus in order. A major simplification is achieved when stellar rotation and stellar magnetism are ignored. Indeed, whenever the accompanying Coriolis, centrifugal and Lorentz forces are neglected, spherical symmetry occurs. Moreover, from a physical viewpoint, rotation and magnetism introduce a multitude of flows, waves, and instabilities, with accompanying yet uncalibrated transport processes, each having a complex feedback on the stellar structure as time evolves (Maeder 2009; Mathis 2013). It is then obvious how to simplify the stellar model computations in first instance, namely by ignoring rotation and magnetism. These phenomena are therefore often only considered when 1D models fail to explain particular observational diagnostics.

How appropriate is it to ignore rotation and magnetism when computing stellar models? Both phenomena are closely related and their importance for stellar evolution depends on

$4 \quad$ Aerts, Mathis, Rogers 
the mass of the star. About $10 \%$ of intermediate- and high-mass stars have a detectable stable large-scale structured fossil magnetic field left from their formation and previous convective phases at the current threshold of CaII H \& K absorption lines or spectropolarimetry based on Zeeman splitting (typically a few Gauss, Donati \& Landstreet 2009; Wade et al. 2016). This percentage is in agreement with the broad distribution of the measured spectroscopic projected equatorial surface rotation velocity, $v \sin i$ (e.g., Zorec \& Royer 2012). The physical interpretation is that such stars are born without or with only a thin convective envelope, preventing the creation of a magnetic dynamo in their outer layers. In absence of the latter, they do not experience a strong magnetized wind and therefore do not lose much angular momentum. While stars born with a mass above some $\sim 15 \mathrm{M}_{\odot}$ do experience a considerable radiation-driven wind (Kudritzki \& Puls 2000) and lose angular momentum efficiently, the majority of stars with $1.3 \lesssim M \lesssim 15 \mathrm{M}_{\odot}$ roughly keep their original $J$ received at birth during the main sequence. Aside from these considerations for the stellar envelope, high- and intermediate-mass stars may have a magnetic dynamo in their convective core region. The strength and properties of such interior field cannot be measured directly, as it is shielded by the extended overlying radiative envelope. Indirect evidence of the existence interior fields, with strength above $10^{5}$ Gauss, was deduced from missing dipole modes in Kepler data of red giants (Fuller et al. 2015; Cantiello, Fuller \& Bildsten 2016). If these dipole modes are missing due to suppression by an interior magnetic field in the radiative core, then such field must occur in about half of the progenitor main sequence stars (Stello et al. 2016). However, Mosser et al. (2017) pointed out that the dipole modes of these red giants are not missing, but are rather mixed modes with depressed amplitudes due to damping, invalidating hypotheses made by Fuller et al. (2015) and Cantiello, Fuller \& Bildsten (2016). Irrespective of this controversy, it is a sensible approach to ignore core and surface magnetism for the mass range $1.3 \lesssim M \lesssim 15 \mathrm{M}_{\odot}$ and to focus on rotation as the prime cause of complexity in 1D stellar models (e.g., Georgy et al. 2013) and in asteroseismology (Buysschaert et al. 2018). Additional attention must be given to mass loss for $M \gtrsim 15 \mathrm{M}_{\odot}$.

In contrast, low-mass stars with $M \lesssim 1.3 \mathrm{M}_{\odot}$ are left with a considerable and deep convective envelope after their formation process, resulting in an efficient dynamo, leading to strong cyclic variability. Their (surface) rotation efficiently slows down during the main sequence due to an effective magnetized wind connected with the envelope dynamo, as first recognized from the pioneering work of $\operatorname{Kraft}(1967$, and references therein). This spin-down offers a way of aging low-mass stars observationally (e.g., Barnes 2003).

1.1.1. 1D Stellar Models without Rotation and Magnetism. Even stellar evolution models representing non-rotating non-magnetic stars have major challenges. A critical aspect is the type of energy transport within the various layers inside a star. In the case where energy transport by means of the diffusion of photons is sufficiently efficient, a radiatively stratified layer whose thermal structure is determined by the radiative temperature gradient, will occur. When photons are unable to transport the energy in particular stellar layers, macroscopic convective energy transport takes place. The time scale of such macroscopic motions is negligible compared to the relevant time scales of stellar evolution. Therefore, turbulent convection in stellar models is often assumed to be time-independent and treated by the mixing-length theory of Böhm-Vitense (1958, MLT), although several alternatives exist (e.g., Canuto \& Mazzitelli 1991). MLT assumes that the convective energy transport is taken care of by one single type of large-size eddy with one characteric mean free path, called the mixing length. This parameter, here denoted as $\alpha_{\mathrm{MLT}}$, is usually expressed in local 
MLT: $m$

ixing-length theory

of convection

The mixing length: a free parameter $\alpha_{\mathrm{MLT}}$ representing the characteristic length scale over which convective fluid elements travel before they dissipate in their environment

Overshooting: $\mathrm{t}$ he phenomenon of turbulent convective fluid elements entering a radiative zone over an unknown distance, expressed as a fraction $\alpha_{\mathrm{ov}}$ of the local pressure scale height pressure scale heights, $H_{P}$, and takes typical values between 0.5 and 2.5 , depending on the mass and metalicity of the star (e.g. Viani et al. 2018, for recent evaluations and calibration with respect to the solar value). For low-mass main-sequence stars, $\alpha_{\text {MLT }}$ influences the size of the outer convective envelope. Intermediate-mass stars with $1.3 \lesssim M \lesssim 2 \mathrm{M}_{\odot}$ have a thin convective envelope and stars born with a higher mass mainly have convection in their fully mixed core. The physical circumstances in these stars are very different from those in the Sun, hence $\alpha_{\mathrm{MLT}}$ is essentially uncalibrated for such stars.

The full and instantaneous mixing caused by convection leaves a clear mark on the chemical history of the star. A major unknown in the theory of stellar interiors is the treatment of the physical conditions in the transition layers between a convective and a radiative zone. The thermal structure, as well as the efficiency of chemical mixing and transport of angular momentum in such transition layers are unknown. The size and location of these transition layers are difficult to determine because they depend on the phenomenon of convective "overshooting" or "penetration" as discussed in Section 3.2.4. Due to their turbulent motion and inertia, the fluid elements do not stop abruptly when entering a radiative layer. Their movement continues over some overshoot depth, $\alpha_{\text {ov }}$ (expressed in $H_{P}$; this is $d_{\text {pen }}$ or $d_{\text {over }}$ for penetration or overshooting, as explained in Section 3.2.4). The treatment of the mixing in this overshoot zone extending beyond the Schwarzschild boundary at radial coordinate $r_{\mathrm{cc}}$, here denoted as $D_{\text {mix }}\left(r>r_{\mathrm{cc}}\right)$, is of critical importance because it determines how much fuel takes part in the nuclear reactions. In particular, the amount of helium/carbon at the end of the core hydrogen/helium burning, depends on the structure, hydrodynamics, and mixing efficiency in the overshoot zone. The observational estimation of the properties of the overshoot zone is hence of major importance, because these determine the core mass during the evolution of the star. Convective envelope overshooting (aka undershooting) may occur as well, but its effect on the star's evolution is small compared to the one of core overshooting, although it does affect the surface chemistry.

1.1.2. 1D Stellar Models with Rotation. A large fraction of early-F to O stars are fast rotators (Zorec \& Royer 2012). Observations of such stars, notably surface abundances and color-magnitude diagrams of clusters, triggered the development of stellar evolution models for rotating stars (e.g., Maeder \& Meynet 2000; Brott et al. 2011; Ekström et al. 2012). These usually adopt the approximation of shellular rotation (Zahn 1992), for which the shape of the star is treated in the framework of the Roche model, with the polar and equatorial radii differing maximally by a factor 1.5 at the critical rotation rate $\Omega_{\text {crit }} \equiv$ $\sqrt{8 G M / 27 R_{\mathrm{p}}^{3}}$ with $R_{\mathrm{p}}$ the polar radius of the star.

Rotation produces latitudinal dependence of the radiative flux, of the effective temperature, of the stellar wind, etc. A basic assumption relied upon to compute models of rotating stars is that the angular momentum lost from the outer envelope at each time step due to a stellar wind, implies a change in the angular velocity distribution inside the star. Moreover, as the core contracts and the envelope expands during the evolution, the rotation profile changes. This introduces a myriad of flows and instabilities causing chemicals to mix and angular momentum to get transported in the radiative zones of stars. Extensive theory has been developed to describe rotationally-induced instabilities and their accompanying mixing processes, both in low-mass stars (e.g., Chaboyer, Demarque \& Pinsonneault 1995) and in intermediate- and high-mass stars (e.g., Talon et al. 1997; Pinsonneault 1997; Heger, Langer \& Woosley 2000). These processes are sometimes implemented with free parameters in evolution codes, e.g., representing the level of chemical mixing and/or angular momentum 
transport. The transport processes are subject to considerable uncertainties because they cannot be properly calibrated by classical observations and few have been tested against simulations. With the advent of asteroseismology, the theory can finally be evaluated. As an example, we confront below the theoretical prediction that $\Omega_{\text {core }} / \Omega_{\text {surf }} \in[1,8]$ for main-sequence stars with $1.7 \lesssim M \lesssim 15 \mathrm{M}_{\odot}$ (e.g. Georgy et al. 2013, Figure 7).

\subsection{Classical Observational Constraints for Stellar Interiors}

High-resolution high signal-to-noise spectroscopy is a major classical observational method to evaluate stellar evolution models. Spectroscopy allows estimation of the effective temperature $\left(T_{\text {eff }}\right)$, gravity $(\log g)$, projected surface rotation $v \sin i \equiv \Omega_{\text {surf }} R \sin i$ with $R$ the stellar radius, and photospheric abundances of stars. The relative precisions of these spectroscopic diagnostics, which are widely available for stars in the Milky Way and Magellanic Clouds, are compared with other diagnostics for stellar interiors in Table 1. Estimation of $\left(T_{\text {eff }}, \log g, v \sin i\right)$ depends on spectrum normalization, particularly of hydrogen lines, and suffers from degeneracies. Despite this, relative uncertainties for $T_{\text {eff }}$ may reach the level of $1 \%$ for low-mass stars, but one usually cannot do better than $5 \%$ for high-mass stars due to the limited number of spectral lines. Relative uncertainties for log are worse due to the degeneracies and are therefore not included in Table 1. Estimation of $v \sin i$ only offers indirect information due to the unknowns $\sin i$ and $R$. Moreover, $v \sin i$ is also subject to uncertainties caused by other spectral line broadening phenomena (e.g. Simón-Díaz \& Herrero 2014; Aerts et al. 2014b). Photospheric abundances deliver a powerful spectroscopic constraint and have been used extensively to evaluate stellar evolution theory. Low-mass stars in the solar neighborhood can be analysed differentially with respect to the Sun, such that systematic uncertainties cancel out. For these, the relative errors of $[\mathrm{Fe} / \mathrm{H}]$ may be as low as 1 to $2 \%$ (e.g., Meléndez et al. 2014; Nissen 2015). In the case of high-mass stars, the relative errors for $[\mathrm{C} / \mathrm{H}],[\mathrm{N} / \mathrm{H}],[\mathrm{O} / \mathrm{H}]$, and $[\mathrm{Fe} / \mathrm{H}]$ are typically at least 5 to $10 \%$ (Morel, Hubrig \& Briquet 2008; Przybilla et al. 2013; Martins et al. 2015). A positive correlation between the surface nitrogen abundance and $v \sin i$ has been established for about half of the OB-type stars (e.g., Dufton et al. 2018, for a recent discussion). However, the nitrogen abundance turned out to be uncorrelated with the measured surface rotation frequency, $\Omega_{\text {surf }}$, and magnetic field for a sample of 68 slowly to moderately rotating B-type field stars (Aerts et al. 2014a). Rather, evidence for a weak correlation between the dominant oscillation mode frequency and the measured nitrogen abundance was found, suggesting some sort of pulsational mixing in this sample. Recent 2D hydrodynamical simulations of convectively driven internal gravity waves (IGWs) for a $3 \mathrm{M}_{\odot}$ star indeed predict efficient particle mixing in radiative layers (Rogers \& McElwaine 2017).

Double-lined spectroscopic eclipsing binaries have long been known as excellent calibrators for stellar models. Indeed, spectroscopic and (multicolor) photometric data covering their orbit offer model-independent stellar masses at the level of 1-3\% from the binary motion (Torres, Andersen \& Giménez 2010, for an extensive review based on 95 objects, covering the mass range 0.2 to $27 \mathrm{M}_{\odot}$ ). Kepler space photometry revealed similar potential for non-eclipsing double-lined binaries pulsating with coherent $\mathrm{p}$ modes from the Phase Modulation method (Murphy, Shibahashi \& Bedding 2016). Double-lined eclipsing binaries have also been used to estimate convective core overshooting, suggesting a mass-dependence (Claret \& Torres 2018). However, core overshooting depends strongly on various other stellar parameters and such correlations have not yet been accounted for in binary modeling,
$\Omega_{\text {surf }}$ : The angular surface rotation frequency of a star $\Omega_{\mathrm{env}}$ : The average angular rotation frequency in the stellar envelope

$\Omega_{\text {core }}$ : The average angular rotation frequency in the core region of the star $\Omega_{\text {crit }}$ : The Roche critical angular rotation frequency of the star defined as $\sqrt{8 G M / 27 R_{\mathrm{p}}^{3}}$ with $R_{\mathrm{p}}$ the polar radius

IGWs: Internal Gravity Waves 
Table 1 Observational diagnostics used to calibrate models of stellar interiors and their optimal relative precision. Type of star stands for LM: low mass; IM: intermediate mass; HM: high mass; RG: red giant; all: all masses; IRFM/SED: Infra Red Flux Method or Spectral Energy Distribution; EB/SB2: double-lined eclipsing binaries; PB1/PB2: binaries with one or two pulsating components. We indicate the diagnostic's dependence on stellar atmosphere (A) and/or stellar interior models (I).

\begin{tabular}{|c|c|c|c|c|}
\hline Method & Type of star & Diagnostic & Precision & Model dependence \\
\hline Spectral lines & LM & $T_{\text {eff }}$, abundances & $\sim 1 \%$ & A: medium \\
\hline Spectral lines & $\mathrm{IM}$ & $T_{\text {eff }}$, abundances & $\sim 2 \%$ & A: medium \\
\hline Spectral lines & HM & $T_{\text {eff }}$, abundances & $\sim 5 \%$ & A: medium \\
\hline 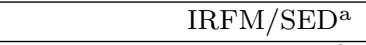 & LM, IM & $T_{\text {eff }}$ & $\sim 2 \%$ & A: low \\
\hline RV \& light curves ${ }^{b}$ & $\mathrm{~EB} / \mathrm{SB} 2$ & $M$ & $\sim 1 \%$ & none \\
\hline RV \& light curves ${ }^{\mathrm{b}}$ & $\mathrm{EB} / \mathrm{SB} 2$ & $R$ & $\sim 3 \%$ & A: low \\
\hline Interferometry $^{\mathrm{c}}$ & all & $R$ & $\sim 3 \%$ & A: low \\
\hline Typical Gaia DR2 ${ }^{\mathrm{d}}$ & LM \& IM & $L$ & $\lesssim 15 \%$ & A: medium \\
\hline Typical Gaia DR2 ${ }^{\mathrm{d}}$ & LM \& IM & $R$ & $\lesssim 10 \%$ & A: medium \\
\hline Cluster (E)MST ${ }^{\mathrm{e}}$ & all & age & $\sim 30 \%$ & I: strong \\
\hline Gyrochronology ${ }^{\mathrm{f}}$ & $\mathrm{LM}$ & $\Omega_{\text {surf }}$ & $\sim 10 \%$ & none \\
\hline Gyrochronology ${ }^{\mathrm{f}}$ & $\mathrm{LM}$ & age & $\sim 20 \%$ & I: medium \\
\hline Coherent g modes ${ }^{\mathrm{g}}$ & $\overline{\mathrm{IM}}$ & $\omega_{n l m}$ & 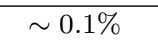 & none \\
\hline Coherent $\mathrm{p}$ modes $\mathrm{g}$ & IM & $\omega_{n l m}$ & $\sim 0.01 \%$ & none \\
\hline Damped p modes ${ }^{g}$ & $\mathrm{LM}$ & $\omega_{n l m}$ & $\sim 0.001 \%$ & none \\
\hline Damped mixed modes ${ }^{g}$ & RG & $\omega_{n l m}$ & $\sim 0.01 \%$ & none \\
\hline g-mode splittings ${ }^{g}$ & IM & $\Omega_{\text {core }}$ & $\sim 0.1 \%$ & none \\
\hline g-mode spacings ${ }^{g}$ & IM & $\Omega_{\text {core }}$ & $\sim 5 \%$ & I: low \\
\hline p-mode splittings $\mathrm{g}$ & $\mathrm{IM}$ & $\Omega_{\mathrm{env}}$ & $\sim 30 \%$ & I: medium \\
\hline p-mode splittings ${ }^{g}$ & LM & $\Omega_{\mathrm{env}}$ & $\sim 50 \%$ & I: medium \\
\hline mixed-mode splittingsg & RG & $\Omega_{\text {core }}$ & $\sim 1 \%$ & none \\
\hline Phase Modulation \& RV ${ }^{g, h}$ & $\mathrm{~PB} 1 / \mathrm{PB} 2$ & $M, R$ & as $\mathrm{EB} / \mathrm{SB} 2$ & none \\
\hline
\end{tabular}

${ }^{a}$ provided that a good estimate of the reddening and an absolute flux calibration are available; this method can also lead to a radius estimate at a level $\sim 3 \%$ when a high-precision parallax (at the level of Gaia DR3) becomes available; ${ }^{b}$ modeling of double-lined eclipsing binaries (EB/SB2) based on radial velocities (RV) from spectroscopy and light curves from photometry, both covering the orbit of the binary (Torres, Andersen \& Giménez 2010); ${ }^{\mathrm{c}}$ requires good calibration stars, as well as a high-precision parallax and $T_{\text {eff }}$, limiting the application to bright stars; ${ }^{d}$ typical values covering 77 million stars (Andrae et al. 2018); ${ }^{e}$ isochrone fitting of (Extended) Main-Sequence Turnoffs, (E)MST, of clusters from combined multi-color photometry, spectroscopy, and astrometry (Bastian \& Lardo 2017); ${ }^{\mathrm{f}}$ from $\mu \mathrm{mag}$ precision time-series space photometry covering many cycles of the rotationally modulated light curve due to spots, delivering $\Omega_{\text {surf }} ;^{\mathrm{g}}$ requires long-duration $\mu$ mag precision uninterrupted space photometric light curves, delivering the frequencies $\omega_{n l m}$ of tens of identified nonradial oscillation modes $(n, l, m)$, where we additionally note that combined $T_{\text {eff }}$, Gaia DR2, and damped modes lead to radii with $\sim 1 \%$ precision for the best cases (Zinn et al. 2018); ${ }^{\mathrm{h}}$ the potential of this method is largest for $\mathrm{p}$ modes and depends on the nature of the pulsating binary (one or two pulsating components), where PB2/SB2 have similar potential as EB/SB2 (cf., Murphy, Shibahashi \& Bedding 2016, for details and first applications).

while this should be done (Johnston et al., submitted).

Interferometric data offers good estimates of angular diameters, translating into precise stellar radii to evaluate stellar models for the very brightest stars. This technique offers a level of precision for $R$ of $\sim 3 \%$ for nearby stars, provided that a good parallax is available 
(e.g. Ligi et al. 2016). Two major limitations with this methods are the limited availability of good calibrators and uncertainties in the limb darkening (e.g. White et al. 2018). In addition to interferometry of bright nearby stars, Gaia astrometry offers the potential to derive accurate radii for millions of stars in the Milky Way, provided that a good estimate of $T_{\text {eff }}$ is available. Gaia DR2 already led to radii at $\sim 2 \%$ precision for a number of (exoplanet host) stars (e.g., Fulton \& Petigura 2018) and even $\sim 1 \%$ for asteroseismic targets (Zinn et al. 2018). At the level of Gaia DR3 (to be released in 2021), stellar radii with a precision of $\sim 1 \%$ are anticipated as the norm for millions of single and binary stars.

Isochrone fitting of color-magnitude diagrams of stellar clusters has been used extensively to test models of stellar interiors, by deducing stellar ages from the observed turnoff point. Large observing campaigns with the Hubble Space Telescope led to systematic detections of multiple main sequences in globular clusters covering masses from 0.1 to $1.8 \mathrm{M} \odot$ and ages from 2 to 15 Gyr (e.g., Piotto et al. 2002; Dotter et al. 2007). These are interpreted as due to different populations of stars with abundance variations indicative of nuclear processing by the CNO cycle (e.g., Piotto et al. 2007; Bastian \& Lardo 2017, for early discoveries and an extensive review, respectively). On the other hand, open clusters with intermediate- and high-mass stars in the Milky Way and Magellanic Clouds reveal extended main sequence turnoffs and/or split main sequences. Interpretation of these in terms of stellar models with rotation leads to ages in agreement with the lithium depletion method. It remains unclear what level of the extended turnoffs is due to rotation (or other phenomena) and what level is due to a spread in age (e.g., Gossage et al. 2018; Bastian et al. 2018). Interpretation of the observed extended turnoffs as solely due to age leads to about $30 \%$ uncertainty for stellar aging. The luminosity $(L)$ estimates deduced from Gaia DR2 data led to spectacular improvements of HR diagrams of clusters (Gaia Collaboration et al. 2018) and Gaia DR3 data is anticipated to bring more potential for the improvement of stellar interiors from fitting of the overall cluster morphologies.

Another way to derive stellar age as an observational test of stellar models is by the method of gyrochronology. Already prior to high-precision space photometry, it was realized that the presence of an envelope dynamo in low-mass stars offers a good way of aging them (Barnes 2003). Gyrochronology is based on the stellar spin-down due to magnetic breaking and uses the surface rotation rate as a clock, adopting the so-called Skumanich relation between angular momentum loss and rotation rate, $d J / d t \propto \Omega_{\text {surf }}^{3}$ (Skumanich 1972). This has meanwhile been extensively applied to Kepler data of low-mass (exoplanet-hosting) field and cluster stars (García et al. 2014; Meibom et al. 2015). Latitudinal surface differential rotation limits gyrochonology for field stars to the level of $\sim 2$ Gyr, i.e., some $20 \%$ relative precision (Epstein \& Pinsonneault 2014). Moreover, the occurrence of anomalously rapid rotation in old field stars hints at weakened magnetic breaking at evolved stages of lowmass stars (van Saders et al. 2016). This implies that more complex models than a simple empirical relation between the rotation rate and stellar age are required for precise stellar aging from gyrochronology of low-mass stars. Such models can be designed by calibrating gyrochronology with asteroseismically determined ages (García et al. 2014; Ceillier et al. 2016). This brings us to the recent breakthroughs in stellar modeling based on asteroseismology, keeping in mind the broad availability of classical diagnostics to evaluate stellar interiors summarized in Table 1. 
Asteroseismology: the interpretation of detected frequencies of identified oscillation modes with radial order, degree and azimuthal order, $(n, l, m)$, in terms of the physical properties of the star's interior

$\omega:$ Angular frequency of an oscillation mode

$N$ : Buoyancy (or Brunt-Väisälä) frequency

\section{THE NEW AGE OF ASTEROSEISMOLOGY}

\subsection{Asteroseismology in a Nutshell}

Stellar oscillations (aka starquakes) offer a direct probe of stellar interiors. Asteroseismology, the interpretation of detected identified oscillation modes (Aerts, Christensen-Dalsgaard \& Kurtz 2010), offers a unique way to evaluate and calibrate stellar interiors. It uses the measured high-precision frequencies of stellar oscillations as revealed by time-domain astronomy as an observational diagnostic of the stellar interior to evaluate stellar models, in addition to classical observables (Table 1). Asteroseismology is the "champion" in the observational probing of stellar interiors.

Asteroseismology follows the foundations laid out for the interpretation of the solar oscillations by means of helioseismology (Christensen-Dalsgaard 2002). The application of this method to distant stars was made possible thanks to uninterrupted high-precision photometric time-series data assembled by recent space missions. In this review, we mainly rely on light curves assembled by the NASA Kepler telescope (Koch et al. 2010) and its refurbished K2 version (Howell et al. 2014). These data have a precision of $\sim \mu$ mag, cover up to four years and three months, respectively, and offered the long awaited evaluation of the theory of stellar interiors. It turns out that shortcomings in the theory of angular momentum transport already occur on the main sequence, impacting all subsequent phases (e.g. Eggenberger et al. 2017; Townsend, Goldstein \& Zweibel 2018; Tayar \& Pinsonneault 2018). As such, time domain space photometry assembled during the last decade is a game changer in the calibration of stellar structure and evolution theories.

Asteroseismic probing of stellar interiors is achieved by interpreting the properties of detected nonradial oscillation modes, notably their frequency. Gravity modes allow one to probe the deepest layers of stars, where chemical gradients are built up during stellar evolution. On the other hand, the $\mathrm{p}$ modes probe the outer envelope of stars. The oscillation modes can often be represented by simple waves, whose angular frequencies $\omega$ fulfill dispersion relations (Smeyers \& Van Hoolst 2010, for a summary of mathematical treatments). As an example, $\mathrm{p}$ modes correspond to acoustic waves and g modes to IGW. For acoustic waves, the dispersion relation involves the sound speed of the gas, while for gravity waves it is the buoyancy (aka Brunt-Väisälä) frequency, $N$. While pressure waves can propagate in both convective and radiative regions, gravity waves can only propagate in convectively stable radiative layers. Just as oscillation modes can be coherent or damped in nature, the corresponding waves can have a standing or traveling wave nature in their propagation cavities inside the star. A broad spectrum of both damped and standing IGW can be generated from partial ionization layers in radiative envelopes and stochastic forcing at convective-radiative interfaces. While the traveling (damped) IGW cannot be used for stellar modeling like their coherent counterparts, unless they coincide with eigenmodes of the star, they do transport angular momentum.

For an extensive discussion on the theory of nonradial oscillation modes, their excitation, their relation to waves and their propagation, we refer to the monographs by Unno et al. (1989) and Aerts, Christensen-Dalsgaard \& Kurtz (2010). The latter book also offers methodology for the frequency analysis and identification of nonradial oscillation modes, in great detail. Here, we limit the discussion to the bare minimum necessary to understand the exploitation of detected oscillation modes and IGW in terms of the stellar interior, with emphasis on rotation. For modern descriptions of coherent mode excitation, we refer to Dupret et al. (2005); Bouabid et al. (2013); Szewczuk \& Daszyńska-Daszkiewicz (2017). 
The plethora of coherent and damped linear oscillation modes inside stars

Whenever a spherically symmetric star in hydrostatic equilibrium gets perturbed, it may become unstable. From a theoretical viewpoint, small disturbances of the equilibrium configuration are treated in a linear approach. As such, the reactions of the acting forces in the equation of motion are investigated to understand and interpret stellar (in)stability (Ledoux 1941; Unno et al. 1989). The instabilities may give rise to detectable self-driven coherent oscillation modes with long lifetimes (thousands to millions of years) whenever their growth rate is positive and when the thermal time scale of the excitation layer in the stellar envelope is longer than the period of the oscillation modes (e.g., Cox 1980; Pamyatnykh 1999). But even when modes are damped, they may still be excited to observable amplitudes by stochastic forcing. This is the case for stars with an outer convective envelope such as the Sun (Christensen-Dalsgaard 2002) and red giants (Chaplin \& Miglio 2013), and gives rise to oscillation modes with lifetimes of days to weeks.

Perturbation of the linearized stellar structure equations leads to the properties of the oscillations of a particular star, among which its mode frequencies. The modes are categorized according to the dominant restoring force. Pressure (or acoustic), gravity, inertial, and magnetic (or Alfvén) oscillations occur when the dominant restoring force is the pressure force, gravity (buoyancy), the Coriolis force, and the Lorentz force, respectively. When stars are hosting close planetary or stellar companions, tidal forces may trigger these different types of modes; in this case they are called tidal oscillations. In practice, the pressure force and gravity are always active, while the other forces may be of minor importance. Hence, one has introduced the specific terminology of $\mathrm{p}$ (for pressure) and $\mathrm{g}$ (for gravity) modes. In reality, the forces act together to restore the equilibrium of the star and one may encounter the situation where two (or more) among them are of similar importance. In that case, the distinction of the modes in terms of frequency may become less clear and the terminology is adapted accordingly, e.g., gravito-inertial modes, magneto-acoustic modes, etc.

IGW generation by convective cores, convective envelopes or thin convection zones due to opacity bumps in radiative envelopes is extensively discussed in Rogers et al. (2013); Talon \& Charbonnel (2008); Cantiello et al. (2009), respectively.

Unlike ground-based time-series data with low duty cycle, high-cadence uninterrupted space photometry does not lead to daily aliasing confusion in the derivation of the oscillation frequencies. As such, the frequency uncertainty is dominated by the frequency resolution of the data set. For coherent modes, this resolution is $\sim 1 / T$, with $T$ the total time base of the time-series data. This is $0.00068 \mathrm{~d}^{-1}(0.0079 \mu \mathrm{Hz})$ for the 4-year nominal Kepler data. For damped modes, the resolution is $\sim 1 / \sqrt{T}$. Aside from the resolution of the data, the frequency error of each detected mode depends on its amplitude and the noise properties of the data in the relevant frequency regime (Aerts, Christensen-Dalsgaard \& Kurtz 2010, Chapter 5). Damped modes have similar amplitudes and allow their frequencies to be deduced from peak bagging (e.g., Lund et al. 2017). Coherent modes, on the other hand, may have mode amplitudes differing six orders of magnitude and their frequencies must therefore be deduced from a prewhitening procedure (e.g., Aerts, Christensen-Dalsgaard \& Kurtz 2010, Chapter 5). Iterative prewhitening inherently introduces uncertainties of order of the frequency resolution of the data. The nominal 4-year Kepler data lead to a relative frequency precision better than $0.1 \%$ for coherent $g$ modes and $0.01 \%$ for coherent $\mathrm{p}$ modes in intermediate- and high-mass stars. Damped p modes of low-mass stars can be measured with a typical relative precision better than $0.001 \%$ for dwarfs (Lund et al. 2017) and 0.01\% 


\section{Spherical harmonics and rotational splitting in asteroseismology}

Following the linear theory of stellar oscillations applied to a spherically symmetric equilibrium star, the displacement vector of a non-radial oscillation mode of degree $l$ and azimuthal order $m$ is given by $\xi(r, \theta, \phi, t)=\left[\left(\xi_{r, n l} e_{r}+\xi_{\mathrm{h}, n l} \nabla_{\mathrm{h}}\right) Y_{l}^{m}(\theta, \phi)\right] \exp (-\mathrm{i} \omega t)$, with $\theta$ the angle measured from the polar axis, $\phi$ the longitude, and $\omega$ the angular mode frequency. In the absence of rotation, the modes are called zonal (for which $m=0$ ); these reveal $l$ surface nodal lines.

In the presence of rotation, the rotation axis of the star is usually chosen as the axis of the spherical polar coordinate system to describe the modes. In this case, $|m|(\leq l)$ of the surface nodal lines are lines of longitude. A distinction is made between prograde $m>0$ and retrograde $m<0$ modes, corresponding with waves travelling with and against the rotation, respectively. The Coriolis acceleration due to the rotation of the star causes rotational splitting of the mode frequencies into $2 l+1$ components called multiplets. The level of splitting among the multiplet components is determined by the rotation frequency $\Omega$ of the star, where larger splitting is due to faster rotation.

The radial order of the mode, $n$, representing the number of nodes of $\xi_{r}$, cannot be deduced from surface observables. Rather, it can be estimated from comparison between observed and theoretically predicted frequencies after identification of $(l, m)$. We refer to the monograph Aerts, Christensen-Dalsgaard \& Kurtz (2010) for detailed methodology to achieve this.

for red giants (Hekker \& Christensen-Dalsgaard 2017; Mosser et al. 2018).

Exploitation of such ultra-precise asteroseismic information has brought a wealth of information on stellar interior processes that is inaccessible from classical data. We dedicate this review to the asteroseismic inference of interior rotation as it constrains internal angular momentum transport. As will be explained below, the asteroseismic estimation of the rotation rate as a function of depth $r$ inside the star, $\Omega(r)$, can be fully or quasi modelindependent. Which of the two it is, depends on the kind of star, the nature of the detected oscillations, and the level of stellar rotation, as explained in Section 2.4. First we discuss how an asteroseismically calibrated model of a star can be sought from its identified modes, before moving on to estimation of the interior rotation.

\subsection{Asteroseismic Modeling of Low-mass Stars}

Asteroseismic modeling relies on 1D spherically symmetric equilibrium models and computations of their 3D eigenmodes and eigenfrequencies by perturbing these $1 \mathrm{D}$ models. For low-mass main-sequence stars, such modeling is based on damped p modes excited by the envelope convection, which reveal themselves with their individual frequencies superposed on a power excess due to granulation (e.g., Kallinger et al. 2014). The p modes have short periods of a few minutes and mainly probe the outer envelope. Given the slow rotation of such stars, their zonal $(m=0)$ p modes can be exploited while neglecting the Coriolis and centrifugal forces. Also the Lorentz force has a negligible effect on the oscillations compared to the pressure force and gravity. The asteroseismic modeling of the zonal-mode frequencies is often based on the same input physics as for the 1D solar model derived from helioseismology (Christensen-Dalsgaard 2002).

Low-degree zonal p modes in low-mass stars reveal a characteristic frequency spacing 
related to the mean density of the star, while the frequency at maximum power is related to the acoustic cut-off frequency depending on $M, R$, and $T_{\text {eff }}$. A spectroscopic measurement of $T_{\text {eff }}$ thus allows to derive $M$ and $R$ with respect to those of the Sun. This potential was predicted long before the space era of asteroseismology in the seminal paper by Kjeldsen \& Bedding (1995) and has by now been applied to hundreds of low-mass dwarfs and subgiants (Chaplin et al. 2014). Once $M$ and $R$ have been derived, a model-dependent stellar age follows. This procedure leads to relative precisions of $\sim 2 \%$ in radius, $\sim 4 \%$ in mass, and $\sim 10 \%$ in age for stars that were monitored during the 4-year nominal Kepler mission and that have similar metalicity and interior physics to the Sun (Silva Aguirre et al. 2017).

Evolved low- and intermediate-mass stars offer, in addition to their p modes, the opportunity to exploit mixed dipole $(l=1)$ modes. These have a p-mode character in the envelope and a g-mode character in the deep interior. In contrast to p modes, g modes have characteristic period spacings determined by the buoyancy frequency $N$. Such spacings were detected from ground-based photometry for white dwarfs long before space asteroseismology (e.g., Kawaler, Sekii \& Gough 1999, for an overview). Red giants reveal mixed modes with gravity-dominated or pressure-dominated character. Evolved stars thus offer both frequency spacings from their $l=0,2 \mathrm{p}$ modes and period spacings from their gravitydominated mixed $l=1$ modes. The potential of mixed modes was studied theoretically by Dupret et al. (2009) and discovered in Kepler data by Beck et al. (2011) and Bedding et al. (2011). The overall probing power of mixed dipole modes allows one to pinpoint the nuclear burning phase of red giants - an assessment that cannot be done from surface measurements (Bedding et al. 2011; Mosser et al. 2014) - but also how the star's core rotates (Beck et al. 2012; Mosser et al. 2012; Deheuvels et al. 2014, cf. Section 2.4). Extensive reviews on 1D red giant modeling based on frequency and period spacings of their damped modes are available in Chaplin \& Miglio (2013) and Hekker \& Christensen-Dalsgaard (2017).

\subsection{Asteroseismic Modeling of Intermediate- and High-mass Stars}

The overall mixing profile beyond the Schwarzschild boundary of the convective core in intermediate-mass and high-mass stars, $D_{\text {mix }}\left(r>r_{\mathrm{cc}}\right)$, is affected by several phenomena, such as convective overshooting/penetration, semi-convection, rotationally- and magnetically-induced instabilities, thermohaline mixing, etc. Theoretically predicted instabilities in the radiatively stratified envelope due to rotation or magnetism lead to discontinuities in $D_{\text {mix }}\left(r>r_{\mathrm{cc}}\right)$. These often remain invisible in evolutionary tracks, but they affect $3 \mathrm{D}$ oscillation mode computations. The latter hence offer a powerful way to calibrate the (free parameters assigned to) mixing and angular momentum transport due to instabilities. In order to achieve that, asteroseismic modeling of an observed star is best done by relying on 1D non-rotating non-magnetic equilibrium models and by computing their mode frequencies. The computation of these $3 \mathrm{D}$ oscillations must take into account the accelerations due to rotation in the perturbed equation of motion. This allows to select the best equilibrium model for the stellar interior and to estimate its free parameters, such as mass, metalicity, and age. Deviations between the predicted and measured oscillation frequencies then reveal shortcomings in the input physics of the equilibrium models and offer a guide to improve it up to the level of precision of the identified oscillation frequencies.

Early asteroseismic modeling of the high-mass B3V star HD 129929 from six identified low-order $l=0,1,2$ modes detected in ground-based data revealed core convective penetration with $\alpha_{\mathrm{ov}}=0.15$ (Aerts et al. 2003; Dupret et al. 2004). In addition, the rotationally 
TAR: Traditional Approximation of Rotation, ignoring the horizontal component of the rotation vector in the equation of motion for a spherically symmetric star split modes led to $\Omega_{\text {core }} / \Omega_{\text {env }} \sim 4$. Following this initial study, major ground-based multi-site campaigns lasting many months and involving tens of astronomers were organised (Handler et al. 2006; Briquet et al. 2012, for some examples). These led to $\alpha_{\mathrm{ov}} \in[0,0.45] \mathrm{H}_{p}$ assuming convective penetration. So far, space missions have observed few high-mass stars and none with suitable identified modes to improve asteroseismic modeling achieved from the ground.

Intermediate-mass stars offer better opportunities, as they reveal tens of low-degree, high-order g modes, with periods of half to a few days. The first detection of g-mode period spacings in main sequence stars came from the CoRoT mission (Degroote et al. 2010) and revealed optimal probing power in the near-core region of stars with a convective core. Such g-mode spacings have meanwhile been detected for a whole range of rotation rates, from very slow to $80 \%$ critical (cf. Section 2.4). Since the g-mode periods may be of similar order to the rotation period, typically 1 to 3 days, the Coriolis force is a mandatory ingredient in the $3 \mathrm{D}$ oscillation computations to interpret the observed period spacing patterns of $\mathrm{g}$ modes, as demonstrated by Van Reeth et al. (2015); Van Reeth, Tkachenko \& Aerts (2016); Ouazzani et al. (2017) for F-type pulsators and by Moravveji et al. (2016); Pápics et al. (2017); Saio et al. (2018b); Szewczuk \& Daszyńska-Daszkiewicz (2018) for B-type pulsators. Unlike for low-mass stars, asteroseismic modeling based on g modes in main sequence stars therefore starts with estimation of the near-core rotation rate $\Omega_{\text {core }}$, as explained in Section 2.4.

Another complication compared to low-mass stars is the need to include physical ingredients that do not occur in the Sun, such as convective overshooting/penetration from the core instead of the envelope. Aerts et al. (2018) and Johnston et al. (submitted) developed a methodological framework to perform asteroseismic modeling of single and binary stars with a convective core, based on tens of identified g modes of consecutive radial order ( $n$ typically between -5 to -50$)$, to estimate $\left(M, X, Z, \Omega_{\text {core }}, D_{\text {mix }}\left(r>r_{\mathrm{cc}}\right), X_{c}\right)$, where $(X, Z)$ are the relative mass fractions of hydrogen and metals such that $X+Y+Z=1$ (with $Y$ the helium mass fraction), and $X_{c}$ is the hydrogen mass fraction in the convective core (a proxy of the age during the main sequence). Their method adopts the so-called Traditional Approximation of rotation (TAR) for the treatment of the Coriolis force (e.g., Townsend $2003 \mathrm{a}, \mathrm{b})$. Estimation of $D_{\text {mix }}\left(r>r_{\mathrm{cc}}\right)$ by g modes is particularly powerful in the core overshoot zone and allows one to derive the core mass of the star (Moravveji et al. 2015, 2016). Further, the modeling required $D_{\text {mix }}\left(r>r_{\mathrm{cc}}\right) \neq 0$ in order to explain the observed g-mode trapping properties, with measured levels of $D_{\text {mix }} \in[1,1000] \mathrm{cm}^{2} \mathrm{~s}^{-1}$. This is smaller than predictions by the theory of rotationally- or magnetically-induced instabilities and provides a new way to calibrate and guide that theory (e.g. Mathis 2013), as well as simulations (Rogers \& McElwaine 2017; Pedersen et al. 2018).

Element and angular momentum transport in stars are intimitely related because they result from the same processes. Often, they are decoupled from each other in stellar evolution codes, where each of the transport phenomena is given its own free parameter because of a lack of transport theories based on first principles. From here on, we focus on angular momentum transport, given that the asteroseismic measurement of $\Omega_{\text {core }}$ is (quasi)independent of $1 \mathrm{D}$ equilibrium models, while estimation of the mixing profile inside the star does depend on it (cf. Table 1). We refer to Salaris \& Cassisi (2017) for a review of the theory of element transport in stars prior to the asteroseismic estimates available today. 


\subsection{Asteroseismic Derivation of Interior Rotation}

The derivation of the interior rotation rates from asteroseismology cannot be done for all pulsators, because it requires the detection and identification of specific oscillation modes. Detection of rotationally split mixed or $\mathrm{g}$ modes is required to derive $\Omega_{\text {core }}$, while split $\mathrm{p}$ modes lead to $\Omega_{\mathrm{env}}$. The approach to follow depends on the ratio of $\Omega$ and $\omega$ and is therefore different for $\mathrm{p}$ and $\mathrm{g}$ modes in different types of stars. Whenever $\Omega / \omega \lesssim 25 \%$, one can rely on a perturbative approach to assess the effects of the Coriolis and centrifugal forces on the stellar oscillations. This is typically the case for $\mathrm{p}$ modes in most stars, for $\mathrm{p}$ and mixed modes in red giants, and for $\mathrm{g}$ modes in subdwarfs and white dwarfs. On the other hand, $\Omega / \omega \gtrsim 25 \%$ requires a non-perturbative treatment of the Coriolis and/or centrifugal forces. Regarding the Coriolis force, this is conveniently done by the TAR for g modes in intermediate- and high-mass stars in the case where $2 \Omega \ll N$.

2.4.1. From a Perturbative Approach. Both the Coriolis and centifugal forces change the mode frequencies. Following a first-order perturbative approach in the computation of the oscillation frequencies is fine when the centrifugal force $\left(\sim \Omega^{2}\right)$ and hence the deformation of the star can be ignored, and when $\Omega \ll \omega$. Let us assume that this is the case and that the rotation rate only changes with depth $r$ inside the star (and not with latitude). Under these assumptions, the mode frequency $\omega_{n l}$ belonging to eigenvector $\xi_{n l}=\left(\xi_{r, n l}, \xi_{\mathrm{h}, n l}\right)$ in the non-rotating case gets split into $2 l+1$ frequency multiplet components due to the Coriolis acceleration. Moreover, each of those components gets shifted due to the Doppler effect according to the rotation between a coordinate system corotating with the star and the inertial coordinate system of the observer. The frequencies of the multiplet in the interial frame then become

$$
\omega_{n l m}=\omega_{n l}+m\left(1-C_{n l}\right) \int_{0}^{R} K_{n l}(r) \Omega(r) \mathrm{d} r
$$

where

$$
K_{n l}(r)=\frac{\left(\xi_{r}{ }^{2}+[l(l+1)] \xi_{\mathrm{h}}^{2}-2 \xi_{r} \xi_{\mathrm{h}}-\xi_{\mathrm{h}}^{2}\right) r^{2} \rho}{\int_{0}^{R}\left(\xi_{r}{ }^{2}+[l(l+1)] \xi_{\mathrm{h}}^{2}-2 \xi_{r} \xi_{\mathrm{h}}-\xi_{\mathrm{h}}^{2}\right) r^{2} \rho \mathrm{d} r},
$$

is called the unimodal rotational kernel and

$$
C_{n l}=\frac{\int_{0}^{R}\left(2 \xi_{r} \xi_{\mathrm{h}}+\xi_{\mathrm{h}}^{2}\right) r^{2} \rho \mathrm{d} r}{\int_{0}^{R}\left(\xi_{r}{ }^{2}+[l(l+1)] \xi_{\mathrm{h}}^{2}\right) r^{2} \rho \mathrm{d} r}
$$

the so-called Ledoux constant (Ledoux 1951). Both $C_{n l}$ and $K_{n l}(r)$ depend on the 1D equilibrium model of the star. However, as outlined in detail in Aerts, Christensen-Dalsgaard $\&$ Kurtz (2010, Chapter 3, Figure 3.38), $C_{n l} \simeq 0$ for high-order or high-degree p modes. On the other hand, the displacement is dominantly horizontal for high-order g modes and the terms containing $\xi_{r}$ in Eqs (2) and (3) can be neglected, leading to

$$
C_{n l} \simeq \frac{1}{[l(l+1)]}
$$

which is independent of the equilibrium model. In the approximation of uniform rotation, the transformation between the corotating frame and the observer's inertial frame implies a frequency shift of $m \Omega$ and one obtains

$$
\omega_{n l m}=\omega_{n l}+m\left(1-C_{n l}\right) \Omega .
$$



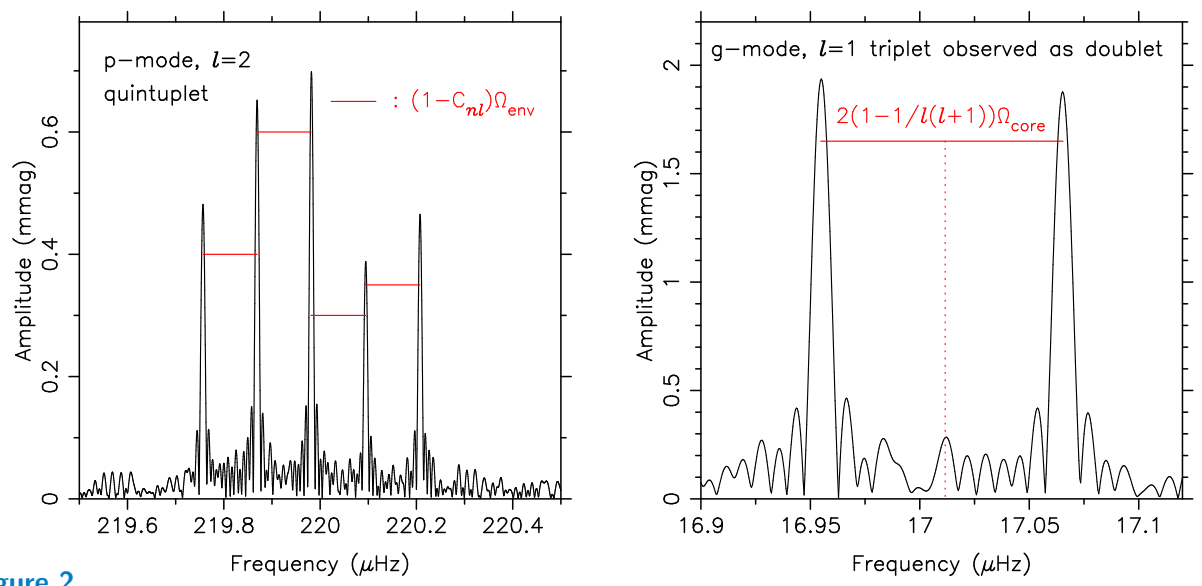

Rotational splitting of a quadrupole p mode (left) and a dipole g-mode (right) of the intermediate-mass star KIC 11145123 . The red dotted line indicates the position of the $(l, m)=(1,0)$ g-mode frequency. Figure produced from data in Kurtz et al. (2014).

In this case the effect of rotation on the oscillation mode frequencies is completely determined by $C_{n l}$ and does not depend on the rotational kernels. For high-order or high-degree p modes, for which $C_{n l} \simeq 0$, the measured rotational splitting between adjacent $m$-values in a multiplet is then given by the average rotation rate in the stellar envelope, $\Omega_{\mathrm{env}}$, in a model-independent way. For low-order p modes, the derivation of $\Omega_{\mathrm{env}}$ depends on the 1D equilibrium model through $C_{n l}$. Depending on the type of star and its evolutionary state, typical values are $C_{n l} \in[0,0.5]$ so the derivation of $\Omega_{\text {env }}$ maximally implies a relative uncertainty of $50 \%$ for $\Omega_{\text {env }}$. Often, however, the model dependence of $C_{n l}$ is negligible, leading to a high-precision quasi model-independent estimate of $\Omega_{\mathrm{env}}$. This is the case for the Kepler target KIC 11145123, whose p-mode splitting is illustrated in the left panel of Figure 2. For high-order g modes, $C_{n l}$ is fully determined by the mode degree, hence their measured splitting offers a model-independent estimate of $\Omega_{\text {core }}$. In particular, the rotational splitting measured from adjacent frequency peaks in a triplet of $\mathrm{g}$ modes equals half the rotation rate. Aside from p modes, KIC 11145123 also shows rotationally split g modes. Given the almost equator-on view upon the stellar rotation axis, its dipole g modes are detected as doublets since the zonal modes almost cancel out in the line-of-sight. This is illustrated in the right panel of Figure 2, where the splitting between the two doublet components offers a direct and model-independent measurement of $\Omega_{\text {core }}$ (Kurtz et al. 2014).

In the general case of differential rotation, the measured rotational splitting is determined by the eigenvector of the mode, $\xi_{n l}$, as well as by the shape of $\Omega(r)$ and the rotational kernels, which depend on the $1 \mathrm{D}$ equilibrium model. In that case, the definition of the corotating frame is not obvious, but a kernel-weighted average of $\Omega(r)$ is a logical choice to compute the Doppler shift towards an inertial frame. As such, one obtains a kernel-weighted average of $\Omega_{\text {env }}$ from p-mode multiplets and a kernel-weighted average of $\Omega_{\text {core }}$ from g modes or from mixed-mode multiplets as detected in Kepler data of red giants (Beck et al. 2012; Mosser et al. 2012).

Perturbation theories up to higher order in $\Omega$ have been developed, e.g., Saio (1981, 2nd order, p modes), Dziembowski \& Goode (1992, 2nd order, p and g modes including magnetism), Lee \& Baraffe (1995, 2nd order, g modes), Soufi, Goupil \& Dziembowski (1998); Daszyńska-Daszkiewicz et al. (2002, 3rd order, p modes), Suárez, Goupil \& Morel 

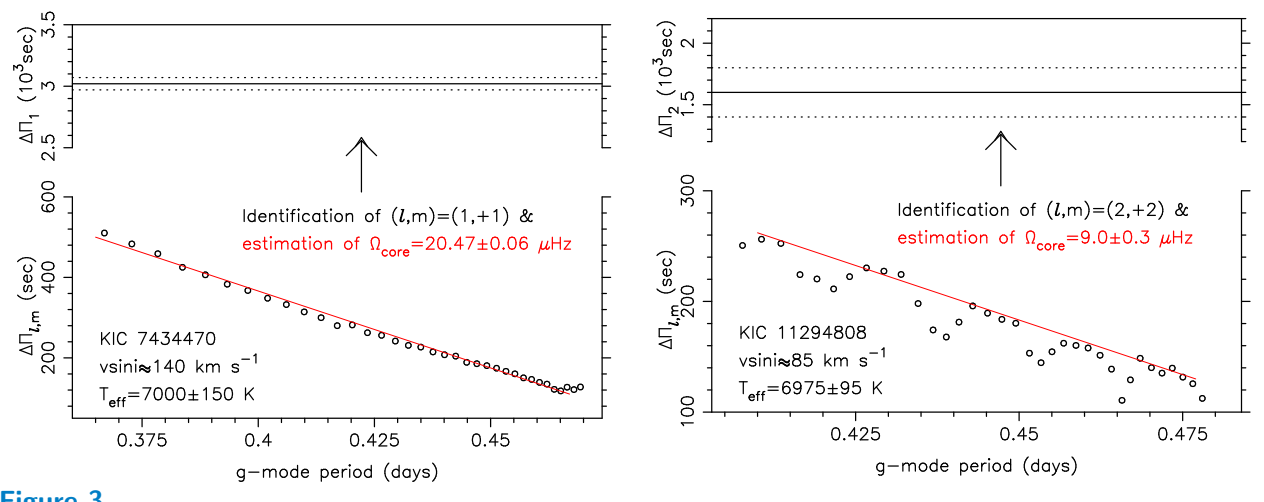

Period spacings versus periods for g modes of consecutive radial order observed in two intermediate-mass F-type stars from 4-year Kepler light curves (circles, errors smaller than the symbol size). These patterns deviate from a constant period spacing that would occur in the absence of rotation. The slope of the observed pattern (red line) is caused by the Coriolis acceleration in the near-core region and allows to derive $\Omega_{\text {core }}$ and to identify dominant $(l, m)$ through the application of Eqs (6) and (8), as indicated by the full lines in the upper panels (dotted lines indicate the uncertainty range for $\Delta \Pi_{l}$ ). The occurrence of mode trapping allows to deduce the star's age and its chemical mixing properties, $D_{\operatorname{mix}}\left(r>r_{\mathrm{cc}}\right)$, after estimation of $\Omega_{\text {core }}$. Figures produced from data in Van Reeth et al. (2015); Van Reeth, Tkachenko \& Aerts (2016).

(2006, 2nd order, p, g, and mixed modes). These theories are based on various assumptions regarding the Coriolis, centrifugal, Lorentz, and tidal forces, and for the ratios of $\Omega, \omega$, the Alfvén frequency $\omega_{\mathrm{A}}$, the orbital frequency of a binary in the oscillation equations and also on the way the deformation of the star $\sim \Omega^{2}$ is treated in the computation of the equilibrium model. Those higher-order perturbation theories leads to particular splittings and shifts in the frequencies of the oscillations compared to $\omega_{n l}$ of the non-rotating case. The theories have in common that they lead to coupling of particular eigenmodes of different $l$ and $m$. The period spacing and mode trapping properties of these various perturbation theories may deviate appreciably from those of non-perturbative theories (cf. Figure 6 in Saio et al. 2018a, for a concrete comparison between TAR and a 2nd order theory for g modes).

2.4.2. Based on the Traditional Approximation. The perturbative approach is no longer appropriate when $\omega$ is of the same order of, or smaller than, $\Omega$. The reason is that the Coriolis acceleration is no longer small compared to the acceleration term in $\omega^{2}$ in the equation of motion. As outlined by Lee \& Saio (1987), the oscillation equations are appreciably simplified when the tangential component of the rotation vector is ignored. This so-called TAR, commonly used in geophysics (Eckart 1960) and in studies of neutron stars (Bildsten, Ushomirsky \& Cutler 1996), allows modes to be computed from the Laplace tidal equations and offers an excellent approximation for g modes in intermediate- and high-mass main sequence stars (see, e.g., Townsend 2003a; Mathis 2013, for comprehensive descriptions), even if they rotate up to a large fraction of their critical rate (Ouazzani et al. 2017).

In a non-rotating star with a convective core, the periods of low-degree, high-order g modes have an asymptotic spacing given by

$$
\Delta \Pi_{l} \equiv \Pi_{l, n}-\Pi_{l, n-1}=\frac{\Pi_{0}}{\sqrt{l(l+1)}},
$$


where

$$
\Pi_{0}=2 \pi^{2}\left(\int_{r_{1}}^{r_{2}} N \frac{\mathrm{d} r}{r}\right)^{-1},
$$

with $r_{1}$ and $r_{2}$ the turning points of $N(r)$, indicative of the boundaries of the convective region(s) that define the g-mode propagation cavity (Tassoul 1980; Smeyers \& Van Hoolst 2010). Typical values for $\Delta \Pi_{1}$ for B-type g-mode pulsators range from $5000 \mathrm{~s}$ to some $12000 \mathrm{~s}$ for $M \in[3,8] \mathrm{M}_{\odot}$ (Degroote et al. 2010; Moravveji et al. 2015). These values decrease as $l$ increases, according to Eq. (6). Van Reeth, Tkachenko \& Aerts (2016) computed $\Delta \Pi_{l}$ for dipole and quadrupole modes of F-type pulsators, varying $\left(M, X, Z, X_{c}, \alpha_{\mathrm{ov}}\right)$ and obtained $\Delta \Pi_{1} \in[2500,3500] \mathrm{s}$ and $\Delta \Pi_{2} \in[1200,2200] \mathrm{s}$ for non-rotating stars.

Inclusion of the Coriolis force in the TAR, assuming uniform rotation, leads to a similar period spacing pattern:

$$
\Delta \Pi_{l, m}=\frac{\Pi_{0}}{\sqrt{\lambda_{l, m, s}}},
$$

with $\lambda_{l, m, S}$ the eigenvalue of the Laplace tidal equation for the g mode with quantum numbers $(l, m)$ and $\mathcal{S}$ the spin parameter $\mathcal{S} \equiv 2 \Omega_{\text {core }} / \omega_{n l m}$. Hence the observed period spacing pattern of a series of $g$ modes with consecutive radial order $n$ and dominant mode numbers $(l, m)$, allows one to simultaneously identify $(l, m)$ and estimate the near-core rotation frequency $\Omega_{\text {core }}$. The procedure is illustrated in Figure 3 for the gravito-inertial modes detected in the Kepler single stars KIC 7434470 and KIC 11294808 (Van Reeth, Tkachenko \& Aerts 2016). From the observed diagnostics, it is found that KIC 7434470 is a $M \simeq 1.4 \mathrm{M}_{\odot}$ star in its early main sequence evolution with $\Omega_{\text {core }} / \Omega_{\text {crit }}=62 \%$, while KIC 11294808 is a $M \simeq 1.9 \mathrm{M}_{\odot}$ star fairly close to the TAMS rotating nearly uniformly $\left(\Omega_{\text {core }} / \Omega_{\mathrm{env}}=98 \%\right.$, Van Reeth et al. 2018$)$ at $59 \%$ of its critical rate (Mombarg et al., in preparation). Their difference in mass and evolutionary stage is not only revealed by the values of $\Delta \Pi_{l}$, but as well by the difference in mode trapping properties: the $\mu$-gradient zone of KIC 11294808 is fairly extensive as its convective core has had a long time to shrink, while KIC 7434470 is in the mass range of having a growing convective core early in its evolution and hence does not reveal mode trapping. In this way, the period spacing patterns such as those in Figure 3 make it possible to estimate $\left(M, X, Z, X_{c}\right)$ after derivation of $\Omega_{\text {core }}$ (Aerts et al. 2018), as well as put constraints on $D_{\text {mix }}\left(r>r_{\mathrm{cc}}\right)$ if mode trapping is observed as for KIC 7434470 in Figure 3. The absence of mode trapping as in the case of KIC 11294808 shown in Figure 3, only allows one to deduce a lower limit of $D_{\text {mix }}\left(r>r_{\mathrm{cc}}\right)$. Asteroseismic modeling of gravito-inertial modes has been put into practice for single and binary B- and F-type g-mode pulsators on the basis of 4-year Kepler light curves by Moravveji et al. (2015, 2016), Van Reeth, Tkachenko \& Aerts (2016), Schmid \& Aerts (2016), Guo, Gies \& Matson (2017), Pápics et al. (2017), Ouazzani et al. (2017), Kallinger et al. (2017), and Szewczuk \& Daszyńska-Daszkiewicz (2018) at various levels of detail.

Mathis (2009) generalized the TAR to include general differential rotation both in radius and latitude. This allows to derive an improved expression compared to the one in Eq. (8) for the period spacing pattern. His formalism was implemented and exploited by Van Reeth et al. (2018) to study the sensitivity of gravito-inertial modes to differential nearcore rotation in a sample of 37 intermediate-mass main sequence stars included in Figure 4 discussed below. It was found that differential rotation can only be measured when period spacing patterns for different degrees $l$ are detected or when the surface rotation rate is measured from rotational modulation or p modes. The stars for which this is fulfilled all have surface-to-core rotation ratios between 0.95 and 1.05. Prat et al. (2017, 2018) go beyond 
the TAR by deriving an asymptotic period spacing for axisymmetric sub-inertial gravitoinertial waves and a general asymptotic theory for gravito-inertial waves propagating within a general differential rotation both in $r$ and $\theta$, respectively. These theoretical advances have yet to be applied to interpret data. Also $2 \mathrm{D}$ oscillation codes have been built to compute oscillations of rapidly rotating stars using either realistic 1D spherical stellar models or 2D deformed, often polytropic, models (e.g. Dintrans \& Rieutord 2000; Reese, Lignières \& Rieutord 2006; Ballot et al. 2010; Ouazzani et al. 2017).

2.4.3. Current Status of Asteroseismic Rotation Rates. The upper panel of Figure 4 shows the asteroseismic estimates of $\Omega_{\text {core }}$ and $\log g$ for 1210 stars available in the literature (dd. 1 August 2018), based on space photometry that led to suitable identified modes allowing to derive these two quantities. The rotation rates were derived as illustrated in Figures 2 and 3 , while the asteroseismic masses and radii were deduced from scaling relations of damped $\mathrm{p}$ modes or from forward modeling of coherent $\mathrm{g}$ modes as discussed in Sections 2.2 and 2.3. These 1210 stars cover the entire evolutionary sequence from core hydrogen burning to the white dwarf remnant phase, for birth masses ranging from 0.72 to $7.9 \mathrm{M}_{\odot}$ and rotation rates from essentially zero up to $80 \%$ of the critical Roche frequency. Several of the main sequence stars are in binaries; these have been indicated as such. While the sample of main sequence stars is not representative in mass, age, rotation rate and binarity, the red clump samples leave no doubt that their helium burning cores have similar angular momentum to subdwarfs and white dwarfs. This result is found for both red clump and secondary clump stars, and is hence independent of having undergone a helium flash or not.

Further, the upper panel of Figure 4 reveales that $\Omega_{\text {core }}$ of all red giants is about two orders of magnitude lower than standard theory of angular momentum transport predicts (e.g. Marques et al. 2013; Goupil et al. 2013; Cantiello et al. 2014; Ouazzani et al. 2018). This means the cores have to lose angular momentum to their overlying envelopes much more efficiently than any current theory predicts. Irrespective of their birth mass or binarity, all red giants reveal that their progenitors are subject to yet unknown physical processes that are able to extract angular momentum from their core in an efficient manner. It has been suggested that the efficiency of the unknown angular momentum transport process(es) is mass dependent (Eggenberger et al. 2017) but it remains unclear if a dichotomy occurs between stars born with and without a convective core (Tayar \& Pinsonneault 2013) or whether the angular momentum transport increases gradually in efficiency with birth mass.

In the lower panel of Figure 4, we shown the 45 stars with a measurement of both $\Omega_{\text {core }}$ and $\Omega_{\text {env }}$ from space photometry. These cover a narrower mass range of $[0.84,3.25] \mathrm{M}_{\odot}$ but an almost equally broad spread in $\Omega / \Omega_{\text {crit }}$ as the upper panel. For some of the measurements in the lower panel, inversion methods similar to those of helioseismology have been used to derive $\Omega(r)$ throughout the star, instead of (kernel-)averaged values for the core and envelope. From those inversion studies, it has been found that the dependence of the results for $\Omega_{\text {core }}$ on the adopted 1D equilibrium models through the use of the rotation kernels is modest and not important in the context of angular momentum transport theory during stellar evolution (see, e.g., Deheuvels et al. (2012, 2014, 2015); Di Mauro et al. (2016); Triana et al. (2017) for sub- and red giants and Triana et al. (2015) for a B-type star). The lower panel of Figure 4 reveals that intermediate-mass main sequence stars rotate nearly uniformly (unless they are member of a binary) and that red clump stars are efficient in reducing the level of differentiality of the rotation acquired during the subgiant and red
Red clump star: a red giant on the horizontal branch that initiated its core-helium burning in degenerate matter through a helium flash $\left(M \lesssim 2.3 \mathrm{M}_{\odot}\right)$

Secondary red clump star: a red giant that initiated its core-helium burning in a non-degenerate state $\left(M \gtrsim 2.3 \mathrm{M}_{\odot}\right)$ 

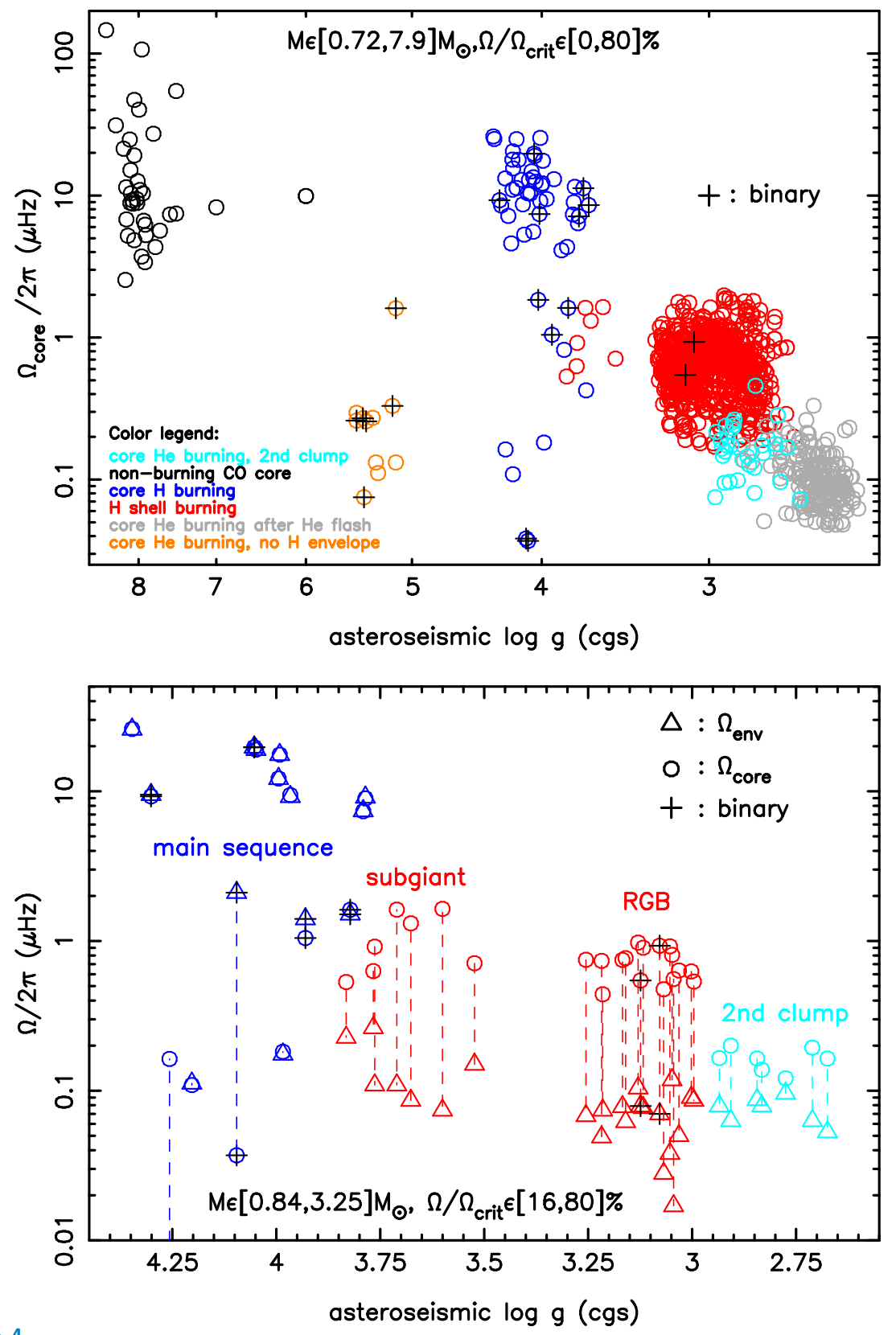

Figure 4

Top: 1210 stars with an asteroseismic estimate of $\Omega_{\text {core }}, M$, and $R$ (dd. 1/8/2018) for the indicated coverage of mass and critical Roche rotation rate as a function of their asteroseismic log $g$, where the latter is a proxy of the evolutionary stage. Typical errors for $\Omega_{\text {core }}$ are listed in Table 1 , while those of $\log g$ range from 0.01 to 0.3 dex and are omitted for optimal visibility. Bottom: 45 stars with an estimate of $\Omega_{\mathrm{env}}$ as well. Figure produced from data in Mosser et al. (2012); Deheuvels et al. (2012, 2014, 2015); Kurtz et al. (2014); Saio et al. (2015); Triana et al. (2015, 2017); Schmid \& Aerts (2016); Murphy, Shibahashi \& Bedding (2016); Moravveji et al. (2016); Di Mauro et al. (2016); Reed (2016); Aerts, Van Reeth \& Tkachenko (2017); Sowicka et al. (2017); Hermes et al. (2017); Guo, Gies \& Matson (2017); Kallinger et al. (2017); Gehan et al. (2018); Buysschaert et al. (2018); Szewczuk \& Daszyńska-Daszkiewicz (2018); Saio et al. (2018a); Beck et al. (2014, 2018); Van Reeth et al. (2018), Johnston et al. (submitted), and Mombarg et al. (in preparation). 
giant phases, when they have a radiative core surrounded by a hydrogen burning shell.

Asteroseismic determination of rotation for main sequence low-mass stars with damped p modes cannot reveal $\Omega_{\text {core }}$, but only $\Omega_{\text {env }}$. By combining their rotationally split $\mathrm{p}$ modes with a high-precision spectroscopic measurement of $v \sin i$ and stellar models or with the frequency of rotational modulation detected in the Kepler data, Benomar et al. (2015) and Nielsen et al. (2017) achieved the envelope and surface rotation rates of 27 such single stars, covering a mass range of 1.0 to $1.6 \mathrm{M}_{\odot}$. This revealed nearly uniform envelope rotation with values $\Omega_{\text {env }} \in[0.4,3.2] \mu \mathrm{Hz}$, in agreement with the spectroscopic $\Omega_{\text {surf }}$ values.

Angular momentum transport by IGW triggered by a convective core in intermediatemass stars (Rogers et al. 2013) and by the envelope in low-mass stars (Charbonnel \& Talon 2005), offers a natural explanation of these observational findings, but there may be other mechanisms as well. In any case, candidate mechanisms must operate and be able to reduce the level of differentiality of $\Omega(r)$ during both core hydrogen and core helium burning for stars with a convective core (Deheuvels et al. 2015; Aerts, Van Reeth \& Tkachenko 2017). Cores of low-mass stars spin up during the subgiant and RGB phases, to reach a level up to ten times the envelope rate, irrespective of whether they belong to a binary or not.

Figure 4 shows $\Omega_{\text {core }}$ rather than the angular momentum of the stellar core, $J_{\text {core }} \equiv$ $M_{\text {core }} \cdot \Omega_{\text {core }} \cdot R_{\text {core }}^{2}$, with $M_{\text {core }}$ and $R_{\text {core }}$ the mass and radius of the stellar core, respectively. The reason is that the asteroseismically derived $\Omega_{\text {core }}$ is (quasi) model-independent, while the computation of $J_{\text {core }}$ requires knowledge of the core mass and core radius, which are model-dependent. A discrepancy of two orders of magnitude between the observed angular momentum of young neutron stars and white dwarfs on the one hand, and the theoretical predictions for $J_{\text {core }}$ of their progenitor stars on the other hand, was previously reported (see Figure 1 in Langer 2012). Asteroseismology has now made it clear that this mismatch must be sought in the early phases of stellar evolution, at least for low- and intermediatemass stars. Their cores lose far more angular momentum than predicted by theory, so an efficient mechanism is required to transport it from the core to the outer envelope. Despite the limited sample of main sequence stars in Figure 4, it is found that the angular momentum transport happens efficiently when the star has a convective core. High-precision asteroseismic measurements of both $\Omega_{\mathrm{env}}$ and $\Omega_{\text {core }}$ from space photometry are currently lacking for high-mass stars, and in particular for blue supergiants. As discussed in the future prospects, this will be remedied in the coming years.

\section{SUMMARY POINTS FROM OBSERVATIONS}

1. Asteroseismology makes it possible to determine the interior rotation of stars, provided that they offer suitable nonradial oscillations to do so; in the cases of rotational splitting detected for mixed or high-order g modes, this is achieved in a model-independent way.

2. Kepler/K2 space photometry of $\mu$ mag precision and 4-year duration led to the core rotation rates of more than 1200 stars covering the mass range $[0.7,8] \mathrm{M}_{\odot}$, rotation frequencies up to $80 \%$ of the critical Roche frequency, and evolutionary phases from core hydrogen burning to the white dwarf remnant phase.

3. Low- and intermediate-mass main sequence stars with an asteroseismic measurement of the core rotation rate, rotate nearly uniformly.

4. Stars lose a large fraction of their initial core angular momentum between the core hydrogen burning phase and the end of core helium burning. 
5. The asteroseismically measured angular momentum of the core of single low- and intermediate-mass stars in the core helium burning phase agrees with the angular momentum of subdwarfs and white dwarfs.

6. Current theory of angular momentum transport falls short by one-to-two orders of magnitude to explain the asteroseismic core rotation rates of evolved low- and intermediate-mass stars.

Armed with these recent asteroseismic constraints on $\Omega(r)$, we now turn our attention to new developments in the theory and simulation of angular momentum transport, offering the reader a historical overview of the relevant facets of this topic and stressing the need for improvements.

\section{ANGULAR MOMENTUM TRANSPORT}

In the standard treatment of stellar structure and evolution, radiative zones are assumed to be motionless. However, as proven by helio- and asteroseismology, they are the seed of dynamical processes, which act on secular time scales to transport angular momentum and mix chemical elements. Here, we focus only on angular momentum transport. Four main processes are identified (Zahn 2013; Mathis 2013): meridional circulation, turbulence driven by instabilities, magnetism, and internal waves. Together these mechanisms give rise to angular momentum transport within radiative interiors of stars. Note, however, that the term "rotational transport/mixing" is sometimes used by the stellar evolution community as terminology for these processes altogether.

\subsection{Hydrodynamical Meridional Circulation: from Eddington Sweet to Zahn's Model}

Historically, the large-scale meridional circulation, which stirs stellar radiative zones, was ascribed to the deformation of the isobars by the centrifugal acceleration, the Lorentz force if there is a magnetic field, and the tidal force if there is a close companion. In that case, the radiative flux is no longer divergence-free (von Zeipel 1924) and should be balanced by heat advection. In the treatment derived by Eddington (1925) and Sweet (1950), the meridional circulation velocity was thus linked to the ratio of the centrifugal acceleration (and the other perturbing forces) to the gravity. The characteristic time scale of this flow was derived by Sweet (1950) and named the Eddington-Sweet time scale: $t_{\mathrm{ES}}=t_{\mathrm{KH}} \frac{G M}{\Omega^{2} R^{3}}$, where $t_{\mathrm{KH}}=\frac{G M^{2}}{R L}$ is the Kelvin-Helmholtz time scale. These results predicted that rapid rotators should be well mixed because of this circulation and should modify their evolution to the giant branch compared to non-rotating stars; this is not observed. This was then improved by Mestel (1953), who accounted for the mean molecular weight (i.e., the $\mu$ gradients), which reduces these circulation effects.

However, the fact that meridional circulation advects angular momentum was ignored. After a first transient phase lasting an Eddington-Sweet time, the star settles into an asymptotic regime. At this stage, the circulation is driven by structural adjustments, torques apply to the star and, when we restrict to the simplest case ignoring fossil magnetic fields and internal waves, internal stresses such as those related to shear-induced turbulence occur 
(Zahn 1992; Rieutord 2006). On one hand, if the star loses angular momentum through a wind, the circulation adjusts to transport angular momentum towards the surface (Zahn 1992; Maeder \& Zahn 1998; Mathis \& Zahn 2004; Decressin et al. 2009). The induced rotation resulting from the advection of the angular momentum is then non-uniform and a baroclinic state sets in, where the temperature varies with latitude along the isobar. On the other hand, if the star does not exchange angular momentum with its environment, the advection by the circulation balances the internal stresses (this is the so-called gyroscopic pumping). In the case of a uniform rotation without any turbulent transport, magnetism, and waves, the circulation thus dies out (Busse 1982). In the case of late-type stars it is hence the loss or gain of angular momentum that drives the circulation, rather than the amplitude of the angular velocity or the related centrifugal acceleration.

The loop of angular momentum transport in stellar radiative zones by large scale circulation can be identified as follows (see Rieutord 2006; Decressin et al. 2009): first, meridional currents are sustained by the torques applied at the stellar surface, by internal stresses such as the viscous ones related to turbulence, and by structural adjustments; next, the temperature relaxes to balance the advection of entropy by the meridional circulation; finally, because of the baroclinic torque induced by the latitudinal distribution of temperature fluctuations on the isobar, a new differential rotation profile is established through the so-called thermal-wind balance. This shear may again generate turbulence, closing the loop.

\subsection{Hydrodynamical Instabilities and Convective Penetration/Overshoot}

Differential rotation in convectively stable radiative zones can induce a large diversity of hydro- and magneto-hydrodynamical instabilities, as discussed below. The turbulence induced by these instabilities can then transport angular momentum. It was already stressed above that mixing at convective-radiative interfaces is critical for both angular momentum and chemical evolution.

The hydrodynamical instabilities, which can take place in the bulk of stellar radiative zones, can be classified in three main families: the Rayleigh-Taylor instability, the baroclinic instabilities, and the shear instabilities. The Rayleigh-Taylor and baroclinic instabilities draw their energy from the potential energy built up by the entropy stratification and the centrifugal acceleration, while the shear instabilities' energy originates from the kinetic energy of the medium.

3.2.1. The Rayleigh-Taylor Instability. The first major instability for a differentially rotating fluid is the so-called Rayleigh-Taylor instability, which is directly linked to the Coriolis acceleration. It was first studied by Lord Rayleigh (1916) in the case of a homogeneous and inviscid flow and by Taylor (1923) in the case of a viscous fluid. Let $s$ be the distance to the axis of rotation in cylindrical coordinates. The so-called Rayleigh or epicyclic frequency $N_{\Omega}$ is defined as

$$
N_{\Omega}^{2}=\frac{1}{s^{3}} \frac{\mathrm{d}}{\mathrm{d} s}\left[\left(s^{2} \Omega\right)^{2}\right]
$$

The differentially rotating fluid is stable if $N_{\Omega}^{2}>0$ and unstable if $N_{\Omega}^{2}<0$. The Rayleigh frequency corresponds to the response time to the restoring Coriolis acceleration and therefore plays an analogous role to the Brunt-Vaïsälä frequency $(N)$ when considering fluctuations in the entropy and chemical stratification. To connect these frequencies, the Brunt-Vaïsälä frequency can conveniently be written as a sum of two frequencies corresponding to the 
restoring of entropy $\left(N_{T}\right)$ and chemical stratification $\left(N_{\mu}\right)$, respectively:

$$
N^{2}=N_{T}^{2}+N_{\mu}^{2}=\frac{g}{H_{P}}\left[\delta\left(\nabla_{\mathrm{ad}}-\nabla\right)+\varphi \nabla_{\mu}\right]
$$

where we have introduced $\nabla=\frac{\partial \ln T}{\partial \ln P}, \nabla_{\text {ad }}=\left(\frac{\partial \ln T}{\partial \ln P}\right)_{S}, \nabla_{\mu}=\frac{\partial \ln \mu}{\partial \ln P}, \delta=\left(\frac{\partial \ln \rho}{\partial \ln T}\right)_{P, \mu}$, and $\varphi=\left(\frac{\partial \ln \rho}{\partial \ln \mu}\right)_{P, T}$, where $\rho$ is the density, $P$ the pressure, $T$ the temperature, $S$ the entropy, and $\mu$ the mean molecular weight. Following Solberg (1936) and Hiland (1941), the Rayleigh criterion can be generalized to take into account the stratification. A differentially rotating stratified region is stable if $N^{2}+N_{\Omega}^{2}>0$ and if the specific angular momentum per unit mass, $j=s \Omega$, grows from the pole towards the equator on each isentropic surface.

3.2.2. Baroclinic Instabilities. In the case of a general differential rotation law rather than uniform or cylindrical rotation $(\Omega(s)$ ), stars are in a baroclinic situation, where the entropy gradient and the gravity are not aligned (e.g. Zahn 1992). This configuration can lead to unstable axisymmetric displacements. The growth of these displacements is hindered by the stable stratification, but this stabilizing effect gets reduced on small scales by thermal diffusivity. Therefore, these instabilities need to be described in the presence of thermal diffusivity $K_{T}$ and viscosity $\nu$, keeping in mind that $K_{T}$ is several orders of magnitude larger than $\nu$. In a stellar radiative region with an unstable differential rotation and a stable entropy stratification, the effect of heat and momentum diffusion is to weaken the effect of stratification by the ratio $\nu / K_{T}$. Such a situation where heat is diffused faster than momentum leads to a so-called double diffusive instability like semi-convection if $N_{T}^{2}<0$ and $N_{\mu}^{2}>0$ and the thermohaline instability if $N_{T}^{2}>0$ and $N_{\mu}^{2}<0$. Goldreich \& Schubert (1967) and Fricke (1968) identified this instability, now known as the Goldreich-SchubertFricke (GSF) instability, and derived the instability criteria:

$$
\frac{\nu}{K_{T}} N_{T}^{2}+N_{\Omega}^{2}<0 \quad \text { or } \quad\left|s \partial_{z} \Omega^{2}\right|>\frac{\nu}{K_{T}} N_{T}^{2},
$$

where $z$ is the coordinate along the rotation axis. The first criterion is the Solberg-Hoiland condition modified by the presence of dissipative processes, while the second one is directly linked to the baroclinicity of the star when $\partial_{z} \Omega \neq 0$. These criteria can be generalized in the case where the chemical stratification is taken into account. The first instability criterion then becomes

$$
\frac{\nu}{K_{T}} N_{T}^{2}+\frac{\nu}{K_{\mu}} N_{\mu}^{2}+N_{\Omega}^{2}<0,
$$

where $K_{\mu}$ is the molecular diffusivity, which is generally of the same order of magnitude as the viscosity. In the case of a strong stabilizing chemical stratification, the GSF instability can be inhibited. However, another axisymmetric instability, the Axisymmetric-BaroClinicDiffusive (ABCD) instability is triggered because of the action of heat diffusion (Knobloch \& Spruit 1983). The instability criterion then becomes

$$
\frac{\nu}{K_{T}}\left(N_{T}^{2}+N_{\mu}^{2}\right)+N_{\Omega}^{2}<0 .
$$

Finally, non-axisymmetric baroclinic instability can be triggered if

$$
\left|\frac{\partial \ln \Omega}{\partial \ln r}\right|>C\left(\frac{N}{\Omega}\right)^{2} \frac{H_{\Omega} \min \left(H_{\rho}, H_{N}, H_{\Omega}\right)}{r^{2}},
$$


where $H_{X}=|\mathrm{d} \ln X / \mathrm{d} r|^{-1}$, with $X=\{\rho, N, \Omega\}$ while $C$ is a coefficient close to unity (Spruit, Knobloch \& Roxburgh 1983; Zahn 1983). This development is favored in weakly stratified regions such as layers close to convective/radiative interfaces.

All the instabilities can play a role in the transport of angular momentum and the mixing of chemicals in stellar interiors (Hirschi \& Maeder 2010). Usually they are implemented as a diffusion coefficient in 1D stellar evolution codes, but their treatment and implementation is challenging since their non-linear saturation is not properly understood nor modeled.

3.2.3. Shear Instability. The second family of hydrodynamical instabilities in stellar radiative zones concerns those triggered by vertical and horizontal shears (Zahn 1992). In a non-stratified case, the necessary condition to have an instability is that the velocity profile $V$ has an inflexion point, i.e., $\mathrm{d}^{2} V / \mathrm{d} x^{2}=0$, where $x$ is the direction along which the instability is considered. In the case of a stably stratified differentially rotating region, the situation becomes more complex (Zahn 1992; Mathis et al. 2018). Indeed, the competition between the destabilizing action of the shear and the stabilizing buoyancy and Coriolis forces has to be examined in detail.

If we first consider the effect of a vertical shear (i.e., along the entropy stratification), assuming as a first step that $2 \Omega \ll N$, the instability is obtained in an adiabatic configuration if $R_{i}=N^{2} / \widehat{\mathcal{S}}^{2}<1 / 4$, where we have introduced the Richardson number $\left(R_{i}\right)$ and the shear $\widehat{\mathcal{S}}=\mathrm{d} V / \mathrm{d} z$. This situation is not reached generally in stellar interiors. However, as in the case of the GSF instability, the action of heat and momentum diffusion should be taken into account. More specifically, since heat is diffused more rapidly than momentum, the heat diffusion weakens the stabilizing action of the entropy stratification. The instability criterion then becomes (e.g. Zahn 1992)

$$
\frac{N^{2}}{\widehat{\mathcal{S}}^{2}} \frac{v \ell}{K_{T}}<R_{i, c},
$$

where $R_{i, c}$ is the critical Richardson number, while $v$ and $\ell$ are characteristic turbulent velocities and length scales, respectively. This leads to the related turbulent transport coefficient along the vertical direction

$$
D_{V, V}=\frac{R_{i, c}}{3} K\left(\frac{r \sin \theta}{N} \frac{\mathrm{d} \Omega}{\mathrm{d} r}\right)^{2},
$$

which has been validated by recent high-resolution direct numerical simulations in a Cartesian geometry (e.g. Prat \& Lignières 2013). From now on $D_{i, j}$ designates the eddy diffusivity corresponding to the turbulent transport along the direction $i$ because of the instability of the shear in the direction $j$. The turbulent motions sustained by the instability of the vertical shear are three-dimensional. As a consequence, they also trigger turbulent transport along the horizontal direction orthogonal to the entropy stratification (Mathis et al. 2018). In stably stratified rotating fluids, the turbulent transport is anisotropic. Indeed, the buoyancy inhibits turbulent motions in the vertical direction while the Coriolis acceleration acts as the restoring force along the horizontal direction. In stellar interiors, where $2 \Omega \ll N$, this leads to a horizontal turbulent transport coefficient derived by Mathis et al. (2018):

$$
D_{H, V}=\frac{N^{4} \tau^{2}}{2 \Omega^{2}} D_{V, V} \quad \text { with generally } \quad D_{H, V} \gg D_{V, V}
$$

where $\tau$ is a characteristic turbulent time scale that depends on the vertical shear and on rotation. We refer to Mathis et al. (2018) for a detailed discussion of this time scale. 
Finally, a horizontal shear (i.e., $\Omega(\theta)$ ) can trigger finite-amplitude instabilities (Zahn 1983; Richard \& Zahn 1999; Dubrulle et al. 2005). This was the first source of turbulence invoked to lead to efficient horizontal transport of momentum and forms the corner stone of the shellular rotation approximation derived by Zahn (1992). Few and incomplete prescriptions have been derived as of today for the related horizontal turbulent transport coefficient $D_{H, H}$. The first one, based on dimensional arguments was proposed by Zahn (1992) but it was found by Maeder (2003) that it leads to configurations along stellar evolution where the condition $D_{V, V} \ll D_{H, H}$ is not respected. Subsequently, Maeder (2003) and Mathis, Palacios \& Zahn (2004) derived two other prescriptions. The first one was based on energetic considerations on the horizontal shear while the second one was using results obtained on the turbulent transport in a non-stratified Couette-Taylor experiment (Richard \& Zahn 1999; Dubrulle et al. 2005). Both prescriptions lead to larger values for $D_{H, H}$ with $D_{V, V} \ll D_{H, H}$ but new theoretical, numerical, and experimental efforts should be pursued to provide a robust ab-initio prediction.

3.2.4. Convective Penetration and Overshoot. A major source of turbulence that should be considered when studying stellar evolution is convective penetration/overshoot at the border(s) of convective/radiative zones. Let us follow Zahn (1991), who provided a coherent physical picture of these processes. First, turbulent stellar convection zones are the seed of large-scale coherent structures commonly referred to as plumes. Because of their inertia, they penetrate into adjacent stellar radiative zones. The key physical control parameter is the Peclet number defined as $P_{e}=v_{c} l_{c} / K_{T}$, where $v_{c}$ and $l_{c}$ are the characteristic convective velocity and length scale, respectively. Two regions are identified. In the first one, $P_{e}>1$, i.e., the flow dynamics is driven by the advection and the plume keeps its identity. The plumes penetrate into the stable region and they render it nearly adiabatic over a penetration distance $d_{\text {pen }}$ while they are decelerated by the buoyancy force. When the Peclet number drops below unity, $P_{e}<1$, the thermal diffusion operates faster than advection and the temperature gradient adjusts from adiabatic to radiative in an overshoot region with thickness $d_{\text {over }}$.

To guide stellar modelers in their quest to describe convective penetration/overshoot, Viallet et al. (2015) proposed three regimes. First, for plumes with $P_{e} \leq 1$, which are only able to mix composition without affecting the entropy structure, an exponential diffusion coefficient as proposed by Freytag, Ludwig \& Steffen (1996) can be adopted. For plumes with $P e \geq 1$, the entropy and the chemicals are both efficiently mixed and one can assume that $\nabla=\nabla_{\text {ad }}$ while the chemical composition is assumed to be mixed instantaneously. Finally for plumes with $P_{e}$. $\gg 1$, turbulent entrainment of mass by convective flows (Fernando 1991) occurs. Another way to improve the quantification of convective penetration and overshoot is by numerical simulations, where strong efforts have been undertaken to follow the whole coverage in Peclet number (e.g. Browning, Brun \& Toomre 2004; Meakin \& Arnett 2007; Viallet et al. 2013; Rogers et al. 2013; Brun et al. 2017).

Nowadays, the overshoot region can be probed observationally by investigating the effect of the different prescriptions on observed and identified oscillation frequencies (e.g. Deheuvels et al. 2016; Constantino, Campbell \& Lattanzio 2017; Pedersen et al. 2018). This is a promising way to constrain the physical properties and free parameters of the prescriptions at the boundaries of convective and radiative layers. Yet another challenging task which asteroseismology may be able to take up in the future is to determine the dependence of convective penetration/overshoot on rotation and magnetic fields. 
Secular 1D stellar modeling has shown that the combined action of hydrodynamical turbulent transport and of the advection by the meridional circulation can explain some properties of massive stars (e.g. Meynet \& Maeder 2000), such as surface abundances (e.g., Hunter et al. 2008). However, as explained in Section 1.2, this is only the case for a fraction of stars. Moreover, since these phenomena are unable to explain the observed rotation profile of the solar radiative interior (e.g. Pinsonneault et al. 1989; Turck-Chièze et al. 2010; Mathis et al. 2018) nor the level of differential rotation of low- and intermediate-mass stars recently found by asteroseismology shown in Figure 4. Therefore, other physical mechanisms must be sought. Magnetic fields and IGW are obvious candidates.

\subsection{Angular Momentum Transport by Magnetism}

As already highlighted in Section 1.2, the magnetic fields of low-mass stars are highly variable dynamo fields, while those observed in about $10 \%$ of the intermediate- and high-mass stars are strong stable structured large-scale fossil fields. A dynamo generated field in the convective core of main sequence stars would not penetrate the extended overlying radiative zone (MacGregor \& Cassinelli 2003). The typical decay time of a fossil magnetic field is given by $\tau_{D} \approx R^{2} / \eta$, where $\eta$ is the magnetic diffusivity. This is approximately $10^{9}$ to $10^{10}$ years, so a fossil magnetic field would be present throughout the stars' evolution, if acted on solely by non-turbulent resistivity.

While the nature and cause of magnetism in stars varies, we are concerned with the angular momentum transport mediated by it, rather than with its origin so we do not discuss the generation mechanism. Moreover, since angular momentum transport within convection zones is efficient due to the turbulent motions (magnetic or otherwise), we focus on angular momentum transport by magnetic fields within radiative regions.

Probably the simplest form of angular momentum transport by magnetic fields comes from the work of Ferraro (1937). Let us start with the magnetic induction equation:

$$
\frac{\partial \mathbf{B}}{\partial t}=\nabla \times(\mathbf{u} \times \mathbf{B})+\eta \nabla^{2} \mathbf{B}
$$

assuming that the magnetic diffusivity $\eta$ is constant. We consider a rotating star with rotation rate $\Omega$ and with a magnetic field $\mathbf{B}$ that is symmetric about the rotation axis. We decompose the magnetic field into poloidal and toroidal components, such that $\mathbf{B}=$ $\mathbf{B}_{T}+\mathbf{B}_{P}$, where $B_{P} \cdot \hat{\phi}=0, B_{T}=B_{\phi} \hat{\phi}$. The only velocity component is that in the azimuthal direction, such that $\mathbf{u}=r \Omega \hat{\phi}$, with $\hat{\phi}$ the unit longitudinal vector. Finally, we neglect magnetic diffusion. The azimuthal component of the magnetic field then evolves according to:

$$
\frac{\partial B_{\phi}}{\partial t}=r\left(B_{p} \cdot \nabla\right) \Omega
$$

Assuming a steady state, this reduces to $\left(B_{p} \cdot \nabla\right) \Omega=0$, which means that $\Omega$ must be constant on poloidal field lines; this is known as Ferraro's iso-rotation law. This homogenization of angular velocity along field lines is mediated by Alfvén waves with a speed $v_{A}=B / \sqrt{\mu_{0} \rho}$, where $\mathrm{B}$ is the magnetic field amplitude and $\mu_{0}$ the permeability of vacuum. Different field lines could rotate at different rates. However, if differential rotation occurred, it would produce a magnetic pressure between field lines, which would exchange angular momentum through phase mixing and eventually these variations in rotation would decay, leaving uniform rotation in the presence of a large scale poloidal field. In the case where the fossil magnetic field connects to adjacent convective zones, some differential rotation 
MRI:

Magneto-Rotational Instability can be transmitted to the radiative zone along the field lines (e.g. Strugarek, Brun \& Zahn 2011). In the case of strong non-axisymmetric fields, rotation is uniform (Spruit 1999).

If we do not consider a steady state, we can immediately see from Eq. (19) that an initially dipolar field will be wound into a toroidal field with its amplitude increasing in time. The presence of such a toroidal field will reduce the efficiency of the angular momentum transfer because the Alfvén waves that mediate angular momentum transfer are diverted azimuthally. Nevertheless, Mestel \& Weiss (1987) found that even a weak magnetic field could impose uniform rotation throughout the star, when considering the solar radiative interior as an example. A similarly weak field would be necessary to impose uniform rotation within the radiative zone of a main sequence star. Of course, the toroidal magnetic field cannot grow indefinitely and is limited by magnetic instabilities.

One expects that the interiors of stars have some degree of radial differential rotation because of the structural adjustments along their evolution and of torques applied at their surface. As discussed in Section 1.1, this is due to the continuous spin down of the stellar surface due to a magnetized wind in the case of low-mass stars and a radiation-driven wind for high-mass stars, while Figure 4 shows that low but significant levels of differentiality are observed for intermediate-mass stars. In such a case, the instabilities that the toroidal components of a magnetic field are likely subject to are the Magneto-Rotational Instability (MRI, Chandrasekhar 1960; Balbus \& Hawley 1992) and/or the Tayler (Tayler 1975)/PittsTayler (Pitts \& Tayler 1985) instability. These instabilities were reviewed by Spruit (1999) under stellar interior conditions.

The MRI occurs in the presence of a weak field when the angular velocity of the system decreases away from the rotation axis, while the angular momentum increases. This instability derives its energy from the differential rotation and has been studied extensively in the astrophysical disks community, where it leads to turbulence within the disk and the requisite angular momentum transfer to allow mass accretion onto the protostar. It has received relatively little attention within the stellar community because the instability is thwarted by the stable stratification. The stability condition in the absence of diffusion is:

$$
q=-\frac{\partial \ln \Omega}{\partial \ln r}>\frac{N^{2}}{2 \Omega^{2}},
$$

where $q$ is the differential rotation parameter. For a typical main sequence star the right hand side is $\sim 10^{5}$, requiring an unrealistically strong radial differential rotation for instability to occur. However, at small scales, the stratification can be offset by thermal diffusion, such that the condition becomes:

$$
q=-\frac{\partial \ln \Omega}{\partial \ln r}>\frac{N^{2}}{2 \Omega^{2}} \frac{\eta}{K_{T}} .
$$

Given that $\eta / K_{T} \ll 1$ in stellar interiors, it is not unreasonable to suppose this instability may proceed in some regions of stellar radiative zones at some ages. Arlt, Hollerbach \& Rudiger (2003) and Jouve, Gastine \& Lignières (2015) studied this instability in spherical shells and both found the instability to occur with consequent increased angular momentum transport. However, both of those studies were carried out for an unstratified gas, hence it is unclear how much those results apply to stellar radiative interiors. The MRI has also been studied within the Sun, where it has been suggested to occur within the tachocline (Kagan \& Wheeler 2014).

Spruit (1999) argued that within stellar interiors, the most likely instability is the Tayler instability. This kink-instability derives its energy from the magnetic field itself and is most 
likely to occur near the poles. The most unstable mode is the $m=1$ mode and the stability condition is:

$$
\frac{\partial \ln \left(B_{T}^{2} \sin \theta \cos \theta\right)}{\partial \theta}>0
$$

which can often be satisfied in the polar regions. The growth rate of the instability depends on rotation and is proportional to $\omega_{A}^{2} / \Omega$, where $\omega_{A}$ is the Alfvén frequency based on the poloidal field strength. Similar to the MRI this instability is limited by stable stratification, which can be offset by thermal diffusion at small scales. Accounting for thermal and chemical stratification, the instability criterion considered by Spruit (1999) is:

$$
\frac{\omega_{A}}{\Omega}>\left(\frac{N^{2}}{\Omega^{2}} \frac{\eta}{\kappa}+\frac{N_{\mu}^{2}}{\Omega^{2}}\right)^{1 / 4}\left(\frac{\eta}{r^{2} \Omega}\right)^{1 / 4}
$$

but a more complete criterion and detailed derivation of it can be found in Zahn, Brun \& Mathis (2007). Building on the robustness of this instability, Spruit (2002) claimed that it could sustain a dynamo in the stably stratified regions of stars. In his description the toroidal field could be generated by differential rotation acting on the initial poloidal field (the standard " $\Omega$ " effect; see Equation 19). Once this toroidal field is sufficiently strong, it becomes unstable to the Tayler instability. The instability then re-produces the latitudinal and radial field components required to close the dynamo loop.

Angular momentum transport by magnetic fields must ultimately be derived from the Lorentz (Laplace) force in the azimuthal component of the momentum equation (Charbonneau \& MacGregor 1993):

$$
\rho r^{2} \sin ^{2} \theta \frac{\partial \Omega}{\partial t}=\frac{1}{\mu_{0}} \mathbf{B}_{P} \cdot \nabla\left(r \sin \theta B_{\phi}\right)+\nabla \cdot\left(\rho \nu r^{2} \sin ^{2} \theta \nabla \Omega\right),
$$

where only magnetic and viscous stresses are taken into account. If one considers variations in radius to be larger than those in latitude, the Maxwell stresses (or magnetic torques) that arise are $\sim B_{r} B_{\phi} / r$. Spruit (2002) assumes that the growth of the field saturates when the generation of the field due to the instability is balanced by the diffusion of the field by turbulent magnetic diffusion. The instability field strength is found by considering the largest scale at which the instability can occur despite the restoring force due to buoyancy and the smallest scale at which the instability still grows in the presence of magnetic diffusion. Accounting for the reduction in the buoyancy force at small scales due to thermal diffusion, this gives an estimate for the instability field strength at saturation. Coupling this to an estimate for the relationship between azimuthal and radial components of the field based on the length scale of the instability, gives an estimate of the Maxwell stress $B_{r} B_{\phi}$, which contributes to angular momentum transport assuming these fields are maximally correlated. Spruit (2002) goes on to assume that this stress can be written as

$$
\mathcal{S}_{\mathrm{m}} \approx \frac{B_{r} B_{\phi}}{4 \pi}=\rho \nu_{e} r \frac{\partial \Omega}{\partial r}
$$

where $\nu_{e}$ is the effective viscosity associated with magnetism. Given the saturation field strengths for $B_{r}$ and $B_{\phi}, \nu_{e}$ can be estimated from the local conditions of the rotation, differential rotation, stratification (both thermal and compositional), and thermal diffusivity. This prescription was implemented into 1D stellar evolution codes (Maeder 2003; Maeder \& Meynet 2004; Heger, Woosley \& Spruit 2005). It was found that this efficient angular momentum transport mechanism could slow down the cores of massive stars sufficiently to 
be more consistent with observations of pulsar rotation rates. In addition, Eggenberger, Maeder \& Meynet (2005) demonstrated it can lead to the uniform rotation observed in the solar radiative core. This was taken as evidence that the so-called Tayler-Spruit dynamo exists and transports angular momentum according to the prescription described above and laid out in Spruit (2002). However, Cantiello et al. (2014) demonstrated that it cannot explain the core rotation of red giants shown in Figure 4.

The arguments laid out by Spruit (2002) were conceptually appealing and could explain some lingering observational questions to do with angular momentum transport. However, there were several physical deficiencies associated with the ansatz, as detailed in Zahn, Brun \& Mathis (2007). The most severe of these is that it remains unclear if a TaylerSpruit dynamo can occur within stellar radiative zones and that, if it did, whether it would saturate as Spruit (2002) envisioned it. Indeed, numerical results by Zahn, Brun \& Mathis (2007) indicate that, while a Tayler instability does occur, it does not produce a dynamo. While small scale radial and latitudinal fields are generated by the instability of the toroidal field, this does not regenerate the large scale axisymmetric poloidal field, which continuously decays in the simulations by Zahn, Brun \& Mathis (2007). Moreover, rather than the instability being saturated by diffusion, it is saturated by the Lorentz (Laplace) force that reacts back on the differential rotation. Therefore, the amplitudes of the fields estimated by Spruit (2002) should be revised. Finally, rather than angular momentum transport behaving as an enhanced viscosity as in Eq. (25), the disturbances to the magnetic field are propagated as Alfvén waves, which do not enhance viscosity. The numerical results by Zahn, Brun \& Mathis (2007) were in conflict with those produced by Braithwaite (2006). Although the latter author claimed to have found a dynamo, his simulations did not include explicit magnetic diffusion and ran less than a magnetic diffusion time, which is insufficiently long. In addition, Braithwaite (2006) permanently sustained differential rotation in his simulations through a body force that does not allow physical back-reaction of the Lorentz force, which generally acts against shear. Recent analytical and numerical work by Goldstein, Townsend \& Zweibel (2018) showed that the occurrence of the Tayler instability may depend on the physical approximation used. We conclude that more work on the Tayler instability in stellar radiative interiors is needed. Despite these limitations of the Spruit ansatz, it remains the de-facto magnetic prescription used in many 1D stellar evolution codes and is used widely throughout the stellar evolution community.

\subsection{Angular Momentum Transport by Internal Gravity Waves}

3.4.1. Wave Generation. Angular momentum transport by IGW depends crucially on the properties of the waves that are generated. Indeed, as discussed in the next section, their propagation and dissipation depend sensitively on their frequency and length scales. Within stars propagative IGW can be generated by any disturbance to the stably-stratified region. The most common and efficient mechanisms of generation are an adjacent turbulent convection zone and tidal forcing by a companion star (or planet).

The tidal generation of waves was discussed in detail in Zahn (1975). The companion causes a disturbance at the convective-radiative interface that generates IGW, which propagate away from the convection zone. Goldreich \& Nicholson (1989) considered the case of intermediate- and high-mass main sequence stars in which the energy of IGW propagates outward. However, the ansatz is equally valid for stars with convective envelopes, in which case the energy of the IGW propagates inward. The waves generated are of large scale 
( $l=2, m=2$ if coplanar, $m=1$ if inclined) and have a frequency equal to the forcing frequency. The dissipation of these waves through critical layers, nonlinear breaking or radiative diffusion (see the next section) can lead to efficient angular momentum exchange between the companion's orbit and the star.

For single stars the predominant source of propagative IGW is their generation by the convective core, although, as we pointed out in Section 2, they can also be generated by a convective envelope or by thin convection zones due to local opacity enhancements in radiative envelopes (the $\kappa$-mechanism). The generation mechanism of IGW has been split into two sources: bulk excitation through Reynolds stresses (e.g. Goldreich \& Kumar 1990) and direct excitation through plumes (e.g. Townsend 1966; Schatzman 1993). The formulation by Goldreich \& Kumar (1990), which was originally developed for the excitation of p modes, has been the most widely adopted version (Kumar \& Quataert 1997; Kumar, Talon \& Zahn 1999; Lecoanet \& Quataert 2013; Shiode et al. 2013), despite the fact that it neglects direct excitation of IGW by plumes, a likely significant source. In that formulation the generation of IGW is treated as an inhomogeneous wave equation with a turbulent source term. Goldreich \& Kumar (1990) argued that the source term was dominated by the quadrupole term (Reynolds stresses) because the monopole (non-adiabatic expansion/contraction of fluid) and dipole (buoyancy) terms, while physically larger, virtually cancel each other. While this assumption and its consequences have been justified in the case of p modes, it is unclear whether it is valid in the case of IGW. It has been demonstrated by Lecoanet et al. (2015) that treating IGW generation as an inhomogeneous wave equation with a source term does reproduce the results of self-consistent, nonlinear numerical simulations of the generation of IGW in the context of laboratory experiments. However, efforts should still be undertaken to formulate the turbulent source term theoretically.

In general, the spectra obtained by the theoretical studies based on the Goldreich \& Kumar (1990) formulation have a steep power-law dependence on frequency. In this formulation the convective turnover (or eddy) frequency is generated with large amplitude, while higher frequencies are generated with significantly lower amplitudes, with the energy in the waves scaling as $E \propto \omega^{-a}$, with $a$ between 3 and 6 . This is in stark contrast with numerical results by Rogers \& MacGregor (2010), Rogers et al. (2013), Alvan, Brun \& Mathis (2014), and Edelmann et al. (2017), who all find power spectra that are much flatter, with frequency dependence $E \propto \omega^{-1}$. This difference has a significant impact on the efficiency with which IGW can transport angular momentum and mix species, where the theoretical results from Goldreich \& Kumar (1990) lead to less efficient transport and mixing than the numerical results. While it is somewhat unclear why there is such a difference between simulations and theory, there are a few obvious contenders. It is unclear whether the approximations made in Goldreich \& Kumar (1990) for p modes are appropriate when applied to IGW, e.g., the source term lacks plume excitation, the turbulent spectra are assumed to be of Kolmogorov type, the effects of rotation and magnetism on turbulence are ignored as is the lack of intermittency of convection. In particular, the theoretical work by Goldreich \& Kumar (1990) relies heavily on the identification of a dominant turnover frequency within the convection zone, but such a frequency does not occur in numerical simulations.

In the theoretical plume model of excitation (e.g. Schatzman 1993), the waves are generated following a Gaussian function in plume size and incursion time, which results in a wave spectrum that is exponential in frequency and wavelength. This model has been revisited by Pinçon et al. (2017, Figure 3), who showed that this process results in a shallow energy generation at low frequency and a steep one at high frequency. Moreover, as 
the plume incursion time is decreased, i.e., as the intrusion becomes more impulsive, the spectrum becomes flatter in frequency, in line with numerical simulations. It appears that theoretical plume models match numerical simulations better than those due to internal stresses (Edelmann et al. 2017), but clearly more work needs to be done.

While the numerical simulations include many of the effects neglected in theoretical work, they lack turbulence corresponding to stellar regimes. Moreover, numerical constraints require enhanced damping of waves. While there is no way to get to realistic levels of turbulence within the convection zone, the simulations tuned to concrete stellar circumstances explain the asteroseismic observations of main sequence stars shown in Figure 4 (Rogers 2015). Some numerical simulations by Rogers et al. (2013) force the convection harder to compensate for enhanced wave damping. While this is not ideal there is no obvious better path forward if the aim is to predict wave amplitudes at the stellar surface. Before we move on to wave propagation and dissipation, we point out that the combined effects of tidally and convectively induced waves has not yet been considered, a lack that should definitely be remedied in the future.

3.4.2. Propagation and Dissipation. In considering the propagation of IGW in stellar interiors, we first discuss propagation of pure IGW, in the absence of rotation and magnetism. In this simplest case IGW propagate in stably stratified regions when $\omega<N$. Whenever waves have high frequency $\omega$ close to $N$, the waves set up standing modes due to internal reflection (Alvan et al. 2015). However, here we focus on low frequency travelling waves, which propagate throughout the interior and are damped before internal reflection. In the limit $\omega \ll N$, the waves have high radial order, which results in the vertical wavelength $\left(\lambda_{v}\right)$ being much smaller than the horizontal one $\left(\lambda_{h}\right)$. In that case, the horizontal velocities $\left(v_{h}\right)$ are much larger than the vertical velocities $\left(v_{v}\right)$, i.e. $\lambda_{v} / \lambda_{h} \sim \omega / N \sim v_{v} / v_{h}$.

There are three main ways to dissipate IGW: 1) linear thermal diffusion, 2) occurrence of critical layers and 3) nonlinear breaking. Thermal diffusion depends sensitively on the wavelength and frequency of the waves, with the damping length in the low-frequency regime $l_{d} \propto K_{T}^{-1} k_{h}^{-3} \omega^{4} N^{-3}$. Critical layers occur when the frequency of the wave is the same as the local rotation frequency. Formally, at such layers, the vertical wavelength goes to zero. However, nonlinear effects become important and the wave effectively breaks, with a damping rate proportional to the square root of the local Richardson number (see 3.2.3). Finally, there are two potential sources of nonlinear wave breaking: overturning of the stratification (convective instability) and shear of the wave itself (Kelvin-Helmholtz instability Thorpe 2018). In stellar interiors, convective instability is likely to happen only in regions where $N$ approaches 0 , such as at the center of low-mass stars and near convectiveradiative interfaces. Therefore, in the bulk of stellar interiors, nonlinear wave breaking would take the form of a Kelvin-Helmholtz instability (Press 1981). The simplest criterion for this type of breaking is provided by $\varepsilon \equiv v_{h} /\left(\lambda_{h} \omega\right) \sim 1$. In intermediate- and high-mass stars, waves generated at the convective core interface may reach sufficient amplitude to satisfy this criterion.

In the case of rotating stars, waves are modified because of the Doppler effect that creates a systematic frequency difference between prograde and retrograde waves. This leads to thermal damping acting differently on prograde and retrograde waves, thus sustaining a net transport of angular momentum. In the case of rapidly rotating stars, waves with frequencies close to $2 \Omega$ are influenced by the Coriolis acceleration. In the case of sub-inertial waves for which $\omega<2 \Omega$ (blue region in Figure 5) the propagation domain is restricted to 


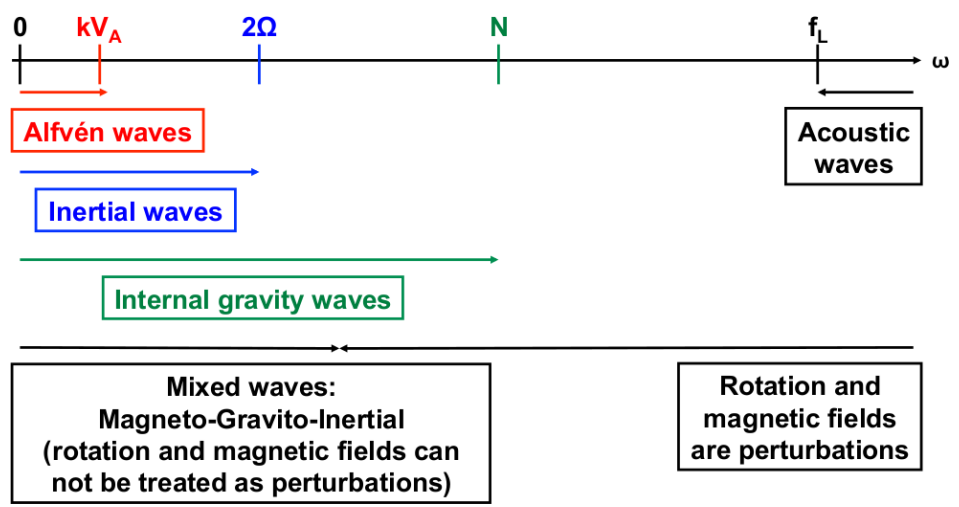

Figure 5

Wave types in a rotating and magnetized stably stratified radiative zone, with associated frequencies; $f_{L}$ is the Lamb frequency and $V_{A}$ the Alfvén speed, while $k$ is the wave vector.

the equatorial region. In the case of super-inertial waves for which $\omega>2 \Omega$ (green region in Figure 5) the waves continue to propagate in the full spherical shell as in the non-rotating case since waves are less influenced by the Coriolis acceleration (Lee \& Saio 1987; Dintrans $\&$ Rieutord 2000). In the case of differential rotation, the behavior of waves is the same but with much more complex propagation cavities than shown schematically in Figure 5 (Prat et al. 2018). This can have important consequences for the transmission of energy from convective to radiative regions and on the transport of angular momentum (Mathis 2009). In the sub-inertial regime, wave-induced transport of angular momentum will be confined to the equatorial region.

Magnetic fields also affect the propagation and dissipation of IGW in stellar interiors. If the field is strong enough such that $\omega_{A}=B /\left(r \sin \theta \sqrt{\mu_{0} \rho}\right) \sim \omega$, IGW can become trapped (e.g., Rogers \& MacGregor 2010; Mathis \& de Brye 2011). In the case of a toroidal field at the convective-radiative interface IGW propagation can be completely blocked, while in the case of a poloidal field IGW are trapped along poloidal field lines. Clearly, this affects low frequency waves more profoundly than the high frequency standing modes used to diagnose stellar interiors. These low frequency waves are the most relevant for the transport of angular momentum.

3.4.3. Transport. In the absence of meridional flows and magnetic fields, the transport of angular momentum by waves is given by a horizontal average of the azimuthal component of the momentum equation (Zahn, Talon \& Matias 1997):

$$
\rho \frac{\mathrm{d}}{\mathrm{d} t}\left(r^{2} \bar{\Omega}\right)=-\frac{1}{r^{2}} \partial_{r}\left(r^{2} \rho r \overline{\sin \theta v_{r} v_{\phi}}\right)+\frac{1}{r^{2}} \partial_{r}\left(\rho \nu r^{4} \partial_{r} \bar{\Omega}\right)
$$

where $v_{r}$ and $v_{\phi}$ are the radial and azimuthal velocities of the waves, respectively, and overbars denote horizontal averages with $\bar{\Omega}=\int_{0}^{\pi} \Omega \sin ^{3} \theta \mathrm{d} \theta / \int_{0}^{\pi} \sin ^{3} \theta \mathrm{d} \theta$ (Zahn 1992). This equation shows that the mean zonal flow is accelerated/decelerated by the divergence of the horizontally-averaged Reynolds stresses, often called the Eliasen-Palm flux (Eliassen \& Palm 1960) and damped by viscous dissipation.

In a global sense, angular momentum transport by IGW can couple convective and ra- 
diative regions (Talon, Kumar \& Zahn 2002; Rogers et al. 2013; Tayar \& Pinsonneault 2013; Fuller et al. 2014; Rogers 2015; Pinçon et al. 2017). Hence, there is major interest in these waves now that the asteroseismic results in Figure 4 require efficient angular momentum coupling between such regions. The Doppler shift for IGW is

$$
\omega(r)=\omega_{\mathrm{gen}}-m\left[\Omega(r)-\Omega_{\mathrm{gen}}\right]
$$

where $\omega_{\text {gen }}$ and $\Omega_{\text {gen }}$ are the frequency of excitation and the angular velocity at the excitation radius, and $\omega(r)$ and $\Omega(r)$ are the frequency and rotation rate in the local reference frame of the wave (Fuller et al. 2014). In the case of weak differential rotation $\Delta \Omega \equiv \Omega(r)-\Omega_{\text {gen }}<\omega_{\text {gen }} / m$, IGW sustain shear until $\Delta \Omega \geq \omega_{\text {gen }} / m$. In that case of stronger differential rotation, critical layers develop and these reduce the differential rotation until $\Delta \Omega=\omega_{\text {gen }} / \mathrm{m}$. From this we immediately deduce that the largest differential rotation that can be tolerated in the presence of IGW is $\Delta \Omega \sim \omega_{\text {gen }} / m$. For waves generated by turbulent convection in a star, the wave spectrum of $\omega$ and $m$ is unknown, which makes it difficult to estimate this limiting differential rotation.

As an example, consider a main sequence star in which the convective core is rotating faster than its radiative envelope, i.e., $\Delta \Omega<0$. Assuming the convection generates prograde and retrograde waves equivalently, the prograde waves $(m>0)$ will be Doppler shifted to higher frequencies (if $\Delta \Omega<0$ ), making them less susceptible to radiative damping as explained in the previous section. These can hence propagate further into the radiative region, where they deposit positive angular momentum when they dissipate. On the other hand, retrograde waves $(m<0)$ are shifted to lower frequencies (if $\Delta \Omega<0$ ) and dissipate closer to the core, spinning down the region, and, more importantly, transporting less angular momentum because of the large density difference between the regions. The outer regions will hence spin up more than the inner regions will spin down. In this way, the convective core is coupled to the radiative envelope from the outside inward. In that case, the transport enforces weak differential rotation. In conclusion, while waves can couple convective and radiative regions, it is expected that these waves cause some positive or negative differential rotation within the radiative regions of stars. Therefore, as in the case of the advection by meridional flows and of the magnetic stresses, the transport of angular momentum by IGW cannot be modeled as a diffusive process.

3.4.4. Application to stars. The first studies of the transport of angular momentum induced by stochastically-excited IGWs were carried out for the Sun (Schatzman 1993; Kumar \& Quataert 1997; Zahn, Talon \& Matias 1997; Talon \& Charbonnel 2005). Talon \& Charbonnel (2005) showed that IGW can couple the convective envelope and radiative interior as well as bring about the uniform rotation of the radiative solar core. However, more detailed analyses of IGW transport in the solar radiative region (Rogers \& Glatzmaier 2006; Denissenkov, Pinsonneault \& MacGregor 2008) indicated that these waves drive weak radial differential rotation, which would be detectable by helioseismology, yet this is not observed. Therefore, while IGW can couple the convective and radiative regions in the Sun, another mechanism seems necessary to explain the uniform rotation profile of the bulk of the solar radiative interior up to $0.25 R_{\odot}$. It is still unclear whether the core of the Sun rotates at the same rate as the bulk of the radiative interior as both García et al. (2007) and Fossat et al. (2017) show an increased rotation rate in the core.

In intermediate- and high-mass stars, IGW are likely to have more dynamical consequences than in low-mass stars, because the waves propagate into a region of decreasing 

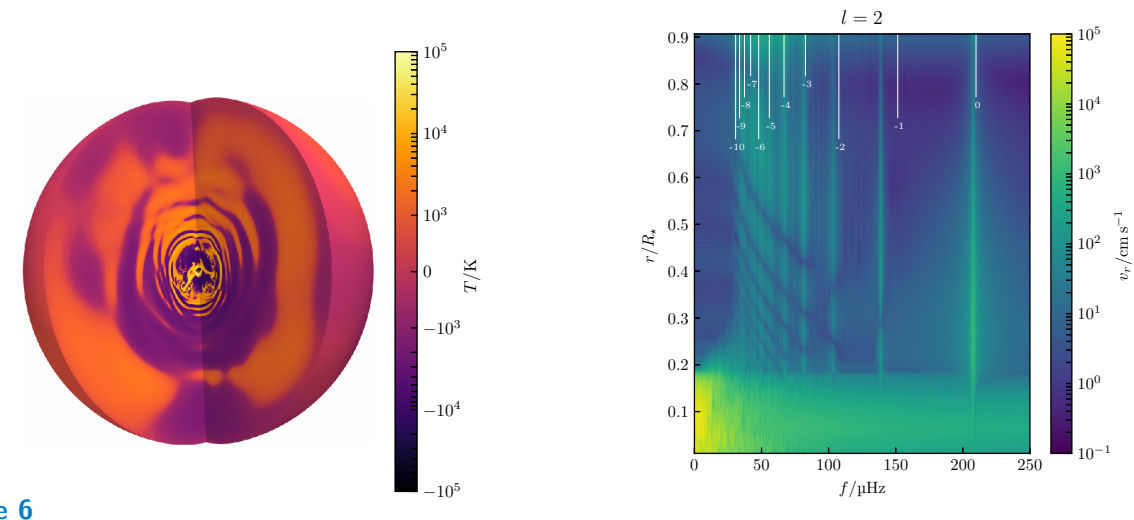

Figure 6

Left: 3D visualization of simulations of IGW from Edelmann et al. (submitted), where the color scale shows temperature fluctuations from the background state. Right: Frequency spectra of the vertical velocity $v_{r}$ for IGW with $l=2$ throughout a $3 \mathrm{M}_{\odot}$ ZAMS star. The vertical white lines are the frequencies of coherent $l=2$ modes of the stellar model for the indicated radial order.

density, allowing the amplitudes of some waves to be amplified. Due to these increased amplitudes, some waves may break and interact with critical layers leading to enhanced angular momentum transport. Moreover, the thermal diffusivity increases rapidly toward the surface of the star, which also leads to efficient damping and angular momentum transport. These effects have been demonstrated in 2D simulations of an equatorial slice for a $3 \mathrm{M}_{\odot}$ main sequence star (Rogers et al. 2013; Rogers 2015). Depending on the strength of the convective flux used for the wave generation and of the level of rotation, several regimes for the resulting differential rotation can be obtained. In cases with high rotation and high forcing, the surface can spin faster than the core in the same direction. In cases with high rotation and low forcing or low rotation and low forcing, uniform rotation is obtained. In cases with low rotation and high forcing, retrograde differential rotation can be driven. Although incomplete in many ways, these numerical results are in agreement with observations of differential rotation in the main sequence stars shown in Figure 4. This was a major motivation to generalize the 2D simulations by Rogers et al. (2013) into 3D, with very similar results in terms of wave spectrum properties and angular momentum transport. A snapshot of the 3D temperature fluctuations is shown in the left panel of Figure 6, while the comparison between the quadrupole component of the generated IGW spectrum and the coherent quadrupole modes is shown on the right (Edelmann et al., submitted). The tangential velocities and temperature fluctuations resulting from these new large-scale long-term simulations are shown in Supplemental Figure 7. Observed frequency values due to coherent $\mathrm{g}$ modes or IGW are dominantly determined by the near-core region for intermediate-mass and high-mass stars, because their amplitudes and hence probing power are dominant there. These fluctuations due to the IGW are currently being used for the generation of synthetic light curves and line-profile variations to evaluate their relevance in explaining low-frequency power excess detected in space photometry (Tkachenko et al. 2014, and Bowman et al. submitted) and signatures of macroturbulence observed in high-mass stars (e.g., Simón-Díaz \& Herrero 2014) for which oscillations have been invoked as a physical explanation (Aerts et al. 2009).

In low-mass evolved stars, the efficiency of the transport of angular momentum driven 
by IGW has been studied for the subgiant phase by Pinçon et al. (2017), who showed that IGWs can extract enough angular momentum to reduce the differential rotation induced by the stellar core contraction. However, IGWs are not efficient enough to explain the weak core rotation of red giants revealed in Figure 4 because of the strong thermal dissipation induced by the high stable stratification in the core (Fuller et al. 2014; Cantiello et al. 2014). As an alternative Belkacem et al. (2015a,b) showed that the angular momentum driven by mixed modes can be a good alternative to explain the observed low rotation rates.

\section{SUMMARY POINTS FROM THEORY AND SIMULATIONS}

1. The zoo of hydrodynamical instabilities occurring in rotating stars is worth revisiting with the aim to calibrate them from asteroseismology; this will lead to a better understanding of their nonlinear properties and behavior as stars evolve.

2. The various theoretical prescriptions of convective penetration and core overshooting can be evaluated from their signature on g modes of single and binary intermediate-mass and high-mass stars; this will lead to an asteroseismically calibrated temperature gradient and mixing profile in the overshoot zone.

3. The theory of angular momentum transport by magnetism based on proper treatment of the Lorentz force should be revisited and included in stellar evolution codes. This can lead to predictions of observables to be tested against asteroseismology of magnetic pulsators of various kind (e.g., Kurtz 1990; Buysschaert et al. 2018).

4. Simulations show that angular momentum transport by IGWs offer great potential to explain the asteroseismic results for $\Omega(r)$ observed in a variety of stars (Figure 4). Strong efforts should be pursued to obtain good predictions for their excitation spectra, their propagation, and their damping in rotating (magnetized) stars.

\section{FUTURE OUTLOOK TO IMPROVE STELLAR MODELING}

Space asteroseismology opened a new window to the Universe, by providing direct measurements of stellar interiors. As such, it revived the theory of stellar structure and evolution, pointing out major challenges for theories that remained uncalibrated so far but have been used in astrophysics for decades. A prominent shortcoming is a proper treatment of angular momentum transport in stellar models.

While the results in Figure 4 are certainly suggestive, a drawback preventing us from deducing a causal connection between the core hydrogen burning stars and their descendants is the lack of an accurate age, aside from the far too small sample sizes. The asteroseismic gravity, while of good precision, is too rough a proxy for the evolutionary status. Therefore, we need to populate Figure 4 with thousands of single and binary stars, covering the premain sequence up to the supergiant phase, with suitable and identified nonradial oscillation modes. Coming back to Figure 1, we have highlighted the major assets and limitations of 1D stellar modeling, keeping in mind the observational diagnostics and their level of precision in Table 1 as well as the current samples in Figure 4.

Improvements in the theory, following a data-driven approach, are within reach from existing Kepler/K2 and Gaia DR2 data. The upcoming Gaia DR3 and TESS data will reveal thousands of suitable pulsators for asteroseismology in the Milky Way and in the Large Magellanic Cloud by the end of 2020. However, probing of $\Omega(r), D_{\operatorname{mix}}(r)$ and other 
quantities in the deep stellar interior requires gravito-inertial or mixed modes and their frequencies can only be deduced from data with a duration of at least one year (Aerts et al. 2018). This can be achieved for stars in the two Continuous Viewing Zones of TESS. On a somewhat longer term, the space data to be assembled with the ESA PLATO mission (Rauer et al. 2014, launch +2026) will deliver the necessary frequency precision for samples of thousands of single and binary stars covering wide ranges in mass, metalicity, and rotation, including pre-main sequence stars and stellar clusters. This big data revolution in stellar astrophysics will not only allow us to populate Figure 4, but also to replace the $\log g$ by a high-precision seismic age estimate and to construct similar diagrams for other quantities of critical importance for stellar interiors, such as the mixing profiles, $D_{\operatorname{mix}}\left(r>r_{\mathrm{cc}}\right)$, inside stars. This revolution will also allow us to finally identify and better model the dominant sources of angular momentum transport.

\section{FUTURE ISSUES}

1. The way forward in stellar modeling is to apply an integrated data-driven approach connecting and developing synergies between observations, new theoretical developments, and new 3D (M)HD simulations according to the spinner in Figure 1. The Kepler/K2 and Gaia data sets largely remain to be exploited with this aim.

2. A theoretical formulation of the optimal turbulent source term for wave generation in a star should be sought.

3. 3D (M)HD simulations covering various nuclear burning phases should be performed; in order for them to be meaningful in terms of diagnostic predictions, these simulations must be global and large-scale in set-up, covering the whole star and have long-duration time bases of several months, so as to cover the observed low-frequency regime observed for stars.

4. An aspect of Figure 1 that remained under-explored is tides in close binaries, the waves they trigger, and their impact on stellar interiors. The integration of tidal theory and binary asteroseismic modeling of observed stars should become a priority.

5. The development of $2 \mathrm{D}$ axisymmetric models taking into account the deformation from spherical symmetry due to the centrifugal force (e.g., Rieutord, Espinosa Lara \& Putigny 2016, ESTER code), is of major importance to model stars rotating close to $\Omega_{\text {crit }}$. Upgrading the current $2 \mathrm{D}$ models by including the chemical evolution of the star, envelope convection, and angular momentum transport is needed to exploit asteroseismic data of the fastest rotators.

6. Current samples of core hydrogen burning and hydrogen shell burning stars with an asteroseismic measurement of $\Omega(r)$ are not representative in terms of mass, rotation, and binarity; those samples need to be extended appreciably to become unbiased.

7. Combined $\mu$ as astrometry, high-resolution high signal-to-noise spectroscopy, and asteroseismic measurements of $\Omega(r)$ and $D_{\operatorname{mix}}(r)$ throughout the star, for single stars, close binaries, and clusters constitutes an optimal route to achieve a better theory of stellar evolution.

8. Asteroseismic measurements of $\Omega(r)$ are hardly available for high-mass stars, and not at all for blue supergiants. The NASA TESS (launched April 2018) and ESA PLATO (to be launched 2026) missions offer the opportunity to assemble the required space photometry and to remedy this lack of stars for both Milky Way and Large Magellanic Cloud populations. 


\section{DISCLOSURE STATEMENT}

The authors are not aware of any affiliations, memberships, funding, or financial holdings that might be perceived as affecting the objectivity of this review.

\section{ACKNOWLEDGMENTS}

The authors dedicate this review to Jean-Paul Zahn, for his education and encouragements to all three of us; his interest and equal respect for all aspects of our research - observations, theory, and simulations - was exceptional in the astrophysics community and of major importance for our careers.

The authors proudly acknowledge the pleasant biannual group discussions and work sessions among their postdoctoral researchers and PhD students the past few years in Leuven, Newcastle, and Saclay, where the research in this review was discussed. They are also grateful for enlightening (email) discussions with Nate Bastian, Paul Beck, Aaron Dotter, Jim Fuller, Thierry Morel, Simon Murphy, Henk Spruit, Andrew Tkachenko, and Rich Townsend. Clio Gielen and Philipp Edelmann are thanked for having produced Figures 1, 6 and 7, and Dominic Bowman, Cole Johnston, Charlotte Gehan, JJ Hermes, Joey Mombarg, Benoit Mosser, May Gade Pedersen, and Timothy Van Reeth for having provided data included in Table 1 and Figures 2 to 4 in electronic format.

The authors received funding from the European Research Council (ERC) under the European Union's Horizon 2020 research and innovation programme (grant agreements No. 670519: MAMSIE with PI Aerts and No. 647383: SPIRE with PI Mathis), from Belspo PLATO grant at KU Leuven, from CNES PLATO grant at CEA/DAp, and from the Science \& Technology Facilities Council (grant agreement ST/L005549/1) as well as from NASA grant NNX17AB92G to PI Rogers.

\section{LITERATURE CITED}

Aerts C, Christensen-Dalsgaard J, Kurtz DW. 2010. Asteroseismology, Astronomy and Astrophysics Library, Springer Berlin Heidelberg

Aerts C, Molenberghs G, Kenward MG, Neiner C. 2014a. Astrophys. J. 781:88

Aerts C, Molenberghs G, Michielsen M, Pedersen MG, Björklund R, et al. 2018. Astrophys. J. Suppl. Ser. 237:15

Aerts C, Puls J, Godart M, Dupret MA. 2009. Astron. Astrophys. 508:409-419

Aerts C, Simón-Díaz S, Groot PJ, Degroote P. 2014b. Astron. Astrophys. 569:A118

Aerts C, Thoul A, Daszyńska J, Scuflaire R, Waelkens C, et al. 2003. Science 300:1926-1928

Aerts C, Van Reeth T, Tkachenko A. 2017. Astrophys. J. Lett. 847:L7

Alvan L, Brun AS, Mathis S. 2014. Astron. Astrophys. 565:A42

Alvan L, Strugarek A, Brun AS, Mathis S, Garcia RA. 2015. Astron. Astrophys. 581:A112

Andrae R, Fouesneau M, Creevey O, Ordenovic C, Mary N, et al. 2018. Astron. Astrophys., in press (arXiv1804.09374)

Arlt R, Hollerbach R, Rudiger G. 2003. Astron. Astrophys. 401:1087-1094

Balbus SA, Hawley JF. 1992. Astrophys. J. 392:662

Ballot J, Lignières F, Reese DR, Rieutord M. 2010. Astron. Astrophys. 518:A30

Barnes SA. 2003. Astrophys. J. 586:464-479 
Bastian N, Kamann S, Cabrera-Ziri I, Georgy C, Ekström S, et al. 2018. MNRAS 480:3739-3746 Bastian N, Lardo C. 2017. Ann. Rev. Astron. Astrophys., in press (arXiv1712.01286)

Beck PG, Bedding TR, Mosser B, Stello D, Garcia RA, et al. 2011. Science 332:205

Beck PG, Hambleton K, Vos J, Kallinger T, Bloemen S, et al. 2014. Astron. Astrophys. 564:A36

Beck PG, Kallinger T, Pavlovski K, Palacios A, Tkachenko A, et al. 2018. Astron. Astrophys. 612:A22

Beck PG, Montalban J, Kallinger T, De Ridder J, Aerts C, et al. 2012. Nat. 481:55-57

Bedding TR, Mosser B, Huber D, Montalbán J, Beck P, et al. 2011. Nat. 471:608-611

Belkacem K, Marques JP, Goupil MJ, Mosser B, Sonoi T, et al. 2015a. Astron. Astrophys. 579:A31

Belkacem K, Marques JP, Goupil MJ, Sonoi T, Ouazzani RM, et al. 2015b. Astron. Astrophys. 579:A30

Benomar O, Takata M, Shibahashi H, Ceillier T, García RA. 2015. MNRAS 452:2654-2674

Bildsten L, Ushomirsky G, Cutler C. 1996. Astrophys. J. 460:827

Böhm-Vitense E. 1958. Zeitschrift für Astrophysik 46:108

Bouabid MP, Dupret MA, Salmon S, Montalbán J, Miglio A, Noels A. 2013. MNRAS 429:2500-2514

Braithwaite J. 2006. Astron. Astrophys. 449:451

Briquet M, Neiner C, Aerts C, Morel T, Mathis S, et al. 2012. MNRAS 427:483-493

Brott I, Evans CJ, Hunter I, de Koter A, Langer N, et al. 2011. Astron. Astrophys. 530:A116

Browning MK, Brun AS, Toomre J. 2004. Astrophys. J. 601:512-529

Brun AS, Strugarek A, Varela J, Matt SP, Augustson KC, et al. 2017. Astrophys. J. 836:192

Busse FH. 1982. Astrophys. J. 259:759-766

Buysschaert B, Aerts C, Bowman DM, Johnston C, Van Reeth T, et al. 2018. Astron. Astrophys. 616:A148

Cantiello M, Fuller J, Bildsten L. 2016. Astrophys. J. 824:14

Cantiello M, Langer N, Brott I, de Koter A, Shore SN, et al. 2009. Astron. Astrophys. 499:279-290

Cantiello M, Mankovich C, Bildsten L, Christensen-Dalsgaard J, Paxton B. 2014. Astrophys. J. 788:93

Canuto VM, Mazzitelli I. 1991. Astrophys. J. 370:295-311

Ceillier T, van Saders J, García RA, Metcalfe TS, Creevey O, et al. 2016. MNRAS 456:119-125

Chaboyer B, Demarque P, Pinsonneault MH. 1995. Astrophys. J. 441:876-885

Chandrasekhar S. 1960. Proceedings of the National Academy of Sciences 46:253-257

Chaplin WJ, Basu S, Huber D, Serenelli A, Casagrande L, et al. 2014. Astrophys. J. Suppl.Ser. 210:1

Chaplin WJ, Miglio A. 2013. Annu. Rev. Astron. Astrophys. 51:353-392

Charbonneau P, MacGregor KB. 1993. Astrophys. J. 417:762

Charbonnel C, Talon S. 2005. Science 309:2189-2191

Christensen-Dalsgaard J. 2002. Rev. of Modern Phys. 74:1073-1129

Claret A, Torres G. 2018. Astrophys. J. 859:100

Constantino T, Campbell SW, Lattanzio JC. 2017. MNRAS 472:4900-4909

Cox JP. 1980. Theory of stellar pulsation, Princeton University Press

Daszyńska-Daszkiewicz J, Dziembowski WA, Pamyatnykh AA, Goupil MJ. 2002. Astron. Astroph. 392:151-159

Decressin T, Mathis S, Palacios A, Siess L, Talon S, et al. 2009. Astron. Astrophys. 495:271-286

Degroote P, Aerts C, Baglin A, Miglio A, Briquet M, et al. 2010. Nat. 464:259-261

Deheuvels S, Ballot J, Beck PG, Mosser B, Østensen R, et al. 2015. Astron. Astrophys. 580:A96

Deheuvels S, Brandão I, Silva Aguirre V, Ballot J, Michel E, et al. 2016. Astron. Astrophys. 589:A93

Deheuvels S, Doğan G, Goupil MJ, Appourchaux T, Benomar O, et al. 2014. Astron. Astrophys. 564:A27

Deheuvels S, García RA, Chaplin WJ, Basu S, Antia HM, et al. 2012. Astrophys. J. 756:19

Denissenkov PA, Pinsonneault M, MacGregor KB. 2008. Astrophys. J. 684:757-769

Di Mauro MP, Ventura R, Cardini D, Stello D, Christensen-Dalsgaard J, et al. 2016. Astrophys. J. 
$817: 65$

Dintrans B, Rieutord M. 2000. Astron. Astrophys. 354:86-98

Donati JF, Landstreet JD. 2009. Ann. Rev. of Astron. Astrophys. 47:333-370

Dotter A, Chaboyer B, Jevremović D, Baron E, Ferguson JW, et al. 2007. Astron. J. 134:376-390

Dubrulle B, Dauchot O, Daviaud F, Longaretti PY, Richard D, Zahn JP. 2005. Physics of Fluids 17:095103-095103

Dufton PL, Thompson A, Crowther PA, Evans CJ, Schneider FRN, et al. 2018. Astron. Astrophys. 615:A101

Dupret MA, Belkacem K, Samadi R, Montalban J, Moreira O, et al. 2009. Astron. Astrophys. 506:57-67

Dupret MA, Grigahcène A, Garrido R, Gabriel M, Scuflaire R. 2005. Astron. Astrophys. 435:927-939

Dupret MA, Thoul A, Scuflaire R, Daszyńska-Daszkiewicz J, Aerts C, et al. 2004. Astron. Astrophys. 415:251-257

Dziembowski WA, Goode PR. 1992. Astrophys. J. 394:670-687

Eckart C. 1960. Physics of Fluids 3:421-427

Eddington AS. 1925. The Observatory 48:73-75

Edelmann P, Roepke F, R.Hirschi, Cyril G, S.Jones. 2017. Astron. Astrophys. 224:245

Edelmann PVF, Röpke FK, Hirschi R, Georgy C, Jones S. 2017. Astron. Astrophys. 604:A25

Eggenberger P, Lagarde N, Miglio A, Montalbán J, Ekström S, et al. 2017. Astron. Astrophys. 599:A18

Eggenberger P, Maeder A, Meynet G. 2005. Astron. Astrophys. 440:L9-L12

Ekström S, Georgy C, Eggenberger P, Meynet G, Mowlavi N, et al. 2012. Astron. Astroph. 537:A146

Eliassen A, Palm E. 1960. Geoph. Pub. 22:1-23

Epstein CR, Pinsonneault MH. 2014. Astrophys. J. 780:159

Fernando HJS. 1991. Ann. Rev. of Fluid Mechanics 23:455-493

Ferraro V. 1937. MNRAS 97:458-473

Fossat E, Boumier P, Corbard T, Provost J, Salabert D, et al. 2017. Astron. Astrophys. 604:A40

Freytag B, Ludwig HG, Steffen M. 1996. Astron. Astrophys. 313:497-516

Fricke K. 1968. Zeitschrift fur Astrophysik 68:317

Fuller J, Cantiello M, Stello D, Garcia RA, Bildsten L. 2015. Science 350:423-426

Fuller J, Lecoanet D, Cantiello M, Brown B. 2014. Astrophys. J. 796:17

Fulton BJ, Petigura EA. 2018. Astron. J., submitted (arXiv1805.01453)

Gaia Collaboration, Babusiaux C, van Leeuwen F, Barstow MA, Jordi C, et al. 2018. Astron. Astrophys., in press (arXiv1804.09378)

García RA, Ceillier T, Salabert D, Mathur S, van Saders JL, et al. 2014. Astron. Astrophys. 572:A34 García RA, Turck-Chièze S, Jiménez-Reyes SJ, Ballot J, Pallé PL, et al. 2007. Science 316:1591

Gehan C, Mosser B, Michel E, Samadi R, Kallinger T. 2018. Astron. Astrophys. 616:A24

Georgy C, Ekström S, Granada A, Meynet G, Mowlavi N, et al. 2013. Astron. Astroph. 553:A24

Goldreich P, Kumar P. 1990. Astrophys. J. 363:694-704

Goldreich P, Nicholson PD. 1989. Astrophys. J. 342:1079-1084

Goldreich P, Schubert G. 1967. Astrophys. J. 150:571

Goldstein J, Townsend RHD, Zweibel EG. 2018. Astrophys. J., submitted (arXiv1808.08958)

Gossage S, Conroy C, Dotter A, Choi J, Rosenfield P, et al. 2018. Astrophys. J. 863:67

Goupil MJ, Mosser B, Marques JP, Ouazzani RM, Belkacem K, et al. 2013. Astron. Astrophys. 549:A75

Guo Z, Gies DR, Matson RA. 2017. Astrophys. J. 851:39

Handler G, Jerzykiewicz M, Rodríguez E, Uytterhoeven K, Amado PJ, et al. 2006. MNRAS 365:327338

Hansen CJ, Kawaler SD, Trimble V. 2004. Stellar interiors: physical principles, structure, and evolution, Astronomy and Astrophysics Library, Springer-Verlag New York

Heger A, Langer N, Woosley SE. 2000. Astrophys. J. 528:368-396 
Heger A, Woosley S, Spruit H. 2005. Astrophys.J. 626:350-363

Hekker S, Christensen-Dalsgaard J. 2017. Astron. Astrophys. Rev. 25:1

Hermes JJ, Gänsicke BT, Kawaler SD, Greiss S, Tremblay PE, et al. 2017. Astrophys. J. Suppl. Ser. 232:23

Hirschi R, Maeder A. 2010. Astron. Astrophys. 519:A16

Howell SB, Sobeck C, Haas M, Still M, Barclay T, et al. 2014. Pub. Astron. Soc. Pac. 126:398

Hunter I, Brott I, Lennon DJ, Langer N, Dufton PL, et al. 2008. Astrophys. J. Lett. 676:L29-L32

Hiland E. 1941. Avhandgliger Norske Videnskaps-Akademi i Oslo, I, Math.-Naturv. Klasse 11:1

Jouve L, Gastine T, Lignières F. 2015. Astron. Astrophys. 575:A106

Kagan D, Wheeler JC. 2014. Astrophys. J. 787:21

Kallinger T, De Ridder J, Hekker S, Mathur S, Mosser B, et al. 2014. Astron. Astrophys. 570:A41

Kallinger T, Weiss WW, Beck PG, Pigulski A, Kuschnig R, et al. 2017. Astron. Astrophys. 603:A13

Kawaler SD, Sekii T, Gough D. 1999. Astrophys. J. 516:349-365

Kippenhahn R, Weigert A, Weiss A. 2012. Stellar Structure and Evolution, Astronomy and Astrophysics Library, Springer Berlin Heidelberg

Kjeldsen H, Bedding TR. 1995. Astron. Astrophys. 293:87-106

Knobloch E, Spruit HC. 1983. Astron. Astrophys. 125:59-68

Koch DG, Borucki WJ, Basri G, Batalha NM, Brown TM, et al. 2010. Astrophys. J. Lett. 713:L79L86

Kraft RP. 1967. Astrophys. J. 150:551

Kudritzki RP, Puls J. 2000. Annu. Rev. Astron. Astrophys. 38:613-666

Kumar P, Quataert E. 1997. Astrophys. J. Lett. 475:133-136

Kumar P, Talon S, Zahn J. 1999. Astrophys. J. 520:859-870

Kurtz DW. 1990. Ann. Rev. Astron. Astrophys. 28:607-655

Kurtz DW, Saio H, Takata M, Shibahashi H, Murphy SJ, Sekii T. 2014. MNRAS 444:102-116

Langer N. 2012. Ann. Rev. Astron. Astrophys. 50:107-164

Lecoanet D, Le Bars M, Burns KJ, Vasil GM, Brown BP, et al. 2015. Physical Review E 91:063016

Lecoanet D, Quataert E. 2013. MNRAS 430:2363-2376

Ledoux P. 1941. Astrophys. J. 94:537

Ledoux P. 1951. Astrophys. J. 114:373

Lee U, Baraffe I. 1995. Astron. Astrophys. 301:419

Lee U, Saio H. 1987. MNRAS 224:513-526

Ligi R, Creevey O, Mourard D, Crida A, Lagrange AM, et al. 2016. Astron. Astrophys. 586:A94

Lund MN, Silva Aguirre V, Davies GR, Chaplin WJ, Christensen-Dalsgaard J, et al. 2017. Astrophys. J. 835:172

MacGregor KB, Cassinelli JP. 2003. Astrophys. J. 586:480-494

Maeder A. 2003. Astron. Astrophys. 399:263-269

Maeder A. 2009. Physics, Formation and Evolution of Rotating Stars, Astronomy and Astrophysics Library, Springer Berlin Heidelberg

Maeder A, Meynet G. 2000. Ann. Rev. Astron. Astrophys. 38:143-190

Maeder A, Meynet G. 2004. Astron. Astrophys. 422:225-237

Maeder A, Zahn JP. 1998. Astron. Astrophys. 334:1000-1006

Marques JP, Goupil MJ, Lebreton Y, Talon S, Palacios A, et al. 2013. Astron. Astrophys. 549:A74

Martins F, Hervé A, Bouret JC, Marcolino W, Wade GA, et al. 2015. Astron. Astrophys. 575:A34

Mathis S. 2009. Astron. Astrophys. 506:811-828

Mathis S. 2013. Transport Processes in Stellar Interiors. In Lecture Notes in Physics, Berlin Springer Verlag, eds. M Goupil, K Belkacem, C Neiner, F Lignières, JJ Green, vol. 865

Mathis S, de Brye N. 2011. Astron. Astrophys. 526:A65

Mathis S, Palacios A, Zahn JP. 2004. Astron. Astrophys. 425:243-247

Mathis S, Prat V, Amard L, Charbonnel C, Palacios A, et al. 2018. Astron. Astrophys., in press (arXiv1808.01814) 
Mathis S, Zahn JP. 2004. Astron. Astrophys. 425:229-242

Meakin CA, Arnett D. 2007. Astrophys. J. 667:448-475

Meibom S, Barnes SA, Platais I, Gilliland RL, Latham DW, Mathieu RD. 2015. Nat. 517:589-591

Meléndez J, Ramírez I, Karakas AI, Yong D, Monroe TR, et al. 2014. Astrophys. J. 791:14

Mestel L. 1953. MNRAS 113:716

Mestel L, Weiss NO. 1987. MNRAS 226:123-135

Meynet G, Maeder A. 2000. Astron. Astrophys. 361:101-120

Moravveji E, Aerts C, Pápics PI, Triana SA, Vandoren B. 2015. Astron. Astrophys. 580:A27

Moravveji E, Townsend RHD, Aerts C, Mathis S. 2016. Astrophys. J. 823:130

Morel T, Hubrig S, Briquet M. 2008. Astron. Astrophys. 481:453-463

Mosser B, Belkacem K, Pinçon C, Takata M, Vrard M, et al. 2017. Astron. Astrophys. 598:A62

Mosser B, Benomar O, Belkacem K, Goupil MJ, Lagarde N, et al. 2014. Astron. Astrophys. 572:L5

Mosser B, Gehan C, Belkacem K, Samadi R, Michel E, Goupil M. 2018. Astron. Astroph., in press (arXiv1807.08301)

Mosser B, Goupil MJ, Belkacem K, Marques JP, Beck PG, et al. 2012. Astron. Astrophys. 548:A10

Murphy SJ, Shibahashi H, Bedding TR. 2016. MNRAS 461:4215-4226

Nielsen MB, Schunker H, Gizon L, Schou J, Ball WH. 2017. Astron. Astroph. 603:A6

Nissen PE. 2015. Astron. Astrophys. 579:A52

Ouazzani RM, Marques JP, Goupil M, Christophe S, Antoci V, Salmon SJAJ. 2018. Astron. Astrophys., submitted (arXiv1801.09228)

Ouazzani RM, Salmon SJAJ, Antoci V, Bedding TR, Murphy SJ, Roxburgh IW. 2017. MNRAS 465:2294-2309

Pamyatnykh AA. 1999. Acta Astron. 49:119-148

Pápics PI, Tkachenko A, Van Reeth T, Aerts C, Moravveji E, et al. 2017. Astron. Astrophys. 598:A74

Pedersen MG, Aerts C, Pápics PI, Rogers TM. 2018. Astron. Astrophys. 614:A128

Pinçon C, Belkacem K, Goupil MJ, Marques JP. 2017. Astron. Astrophys. 605:A31

Pinsonneault M. 1997. Ann. Rev. Astron. Astrophys. 35:557-605

Pinsonneault MH, Kawaler SD, Sofia S, Demarque P. 1989. Astrophys. J. 338:424-452

Piotto G, Bedin LR, Anderson J, King IR, Cassisi S, et al. 2007. Astrophys. J. Lett. 661:L53-L56

Piotto G, King IR, Djorgovski SG, Sosin C, Zoccali M, et al. 2002. Astron. Astrophys. 391:945-965

Pitts E, Tayler R. 1985. MNRAS 216:139-154

Prat V, Lignières F. 2013. Astron. Astrophys. 551:L3

Prat V, Mathis S, Augustson K, Lignières F, Ballot J, et al. 2018. Astron. Astrophys. 615:A106

Prat V, Mathis S, Lignières F, Ballot J, Culpin PM. 2017. Astron. Astrophys. 598:A105

Press WH. 1981. Astrophys. J. 245:286-303

Przybilla N, Nieva MF, Irrgang A, Butler K. 2013. Hot stars and cosmic abundances. vol. 63 of EAS Pub. Ser.

Rauer H, Catala C, Aerts C, Appourchaux T, Benz W, et al. 2014. Exp. Astron. 38:249-330

Rayleigh. 1916. Proc. Roy. Soc. London A 93:148

Reed MD. 2016. IAU Focus Meeting 29:589-595

Reese D, Lignières F, Rieutord M. 2006. Astron. Astrophys. 455:621-637

Richard D, Zahn JP. 1999. Astron. Astrophys. 347:734-738

Rieutord M. 2006. Astron. Astrophys. 451:1025-1036

Rieutord M, Espinosa Lara F, Putigny B. 2016. Journal of Computational Physics 318:277-304

Rogers T, Glatzmaier G. 2006. Astrophys. J. 653:756-764

Rogers TM. 2015. Astrophys. J. Lett. 815:L30

Rogers TM, Lin DNC, McElwaine JN, Lau HHB. 2013. Astrophys. J. 772:21

Rogers TM, MacGregor KB. 2010. MNRAS 401:191-196

Rogers TM, McElwaine JN. 2017. Astrophys. J. Lett. 848:L1

Saio H. 1981. Astrophys. J. 244:299-315 
Saio H, Bedding TR, Kurtz DW, Murphy SJ, Antoci V, et al. 2018a. MNRAS 477:2183-2195

Saio H, Kurtz DW, Murphy SJ, Antoci VL, Lee U. 2018b. MNRAS 474:2774-2786

Saio H, Kurtz DW, Takata M, Shibahashi H, Murphy SJ, et al. 2015. MNRAS 447:3264-3277

Salaris M, Cassisi S. 2017. Roy. Soc. Open Science 4:170192

Schatzman E. 1993. Astron. Astrophys. 279:431-446

Schmid VS, Aerts C. 2016. Astron. Astrophys. 592:A116

Shiode JH, Quataert E, Cantiello M, Bildsten L. 2013. MNRAS 430:1736-1745

Silva Aguirre V, Lund MN, Antia HM, Ball WH, Basu S, et al. 2017. Astrophys. J. 835:173

Simón-Díaz S, Herrero A. 2014. Astron. Astrophys. 562:A135

Skumanich A. 1972. Astrophys. J. 171:565

Smeyers P, Van Hoolst T, eds. 2010. Linear Isentropic Oscillations of Stars: Theoretical Founda-

tions. vol. 371 of Astrophysics and Space Science Library

Solberg H. 1936. Procès Verbaux Ass. Mtor., UGGI, 6e Assemblé Générale, Edinburgh, Mém. et Disc. 2:66

Soufi F, Goupil MJ, Dziembowski WA. 1998. Astron. Astroph. 334:911-924

Sowicka P, Handler G, Dębski B, Jones D, Van de Sande M, Pápics PI. 2017. MNRAS 467:4663-4673

Spruit HC. 1999. Astron. Astrophys. 349:189-202

Spruit HC. 2002. Astron. Astrophys. 381:923-932

Spruit HC, Knobloch E, Roxburgh IW. 1983. Nat. 304:520-522

Stello D, Cantiello M, Fuller J, Huber D, García RA, et al. 2016. Nat. 529:364-367

Strugarek A, Brun AS, Zahn JP. 2011. Astron. Astrophys. 532:A34

Suárez JC, Goupil MJ, Morel P. 2006. Astron. Astrophys. 449:673-685

Sweet PA. 1950. MNRAS 110:548

Szewczuk W, Daszyńska-Daszkiewicz J. 2017. MNRAS 469:13-46

Szewczuk W, Daszyńska-Daszkiewicz J. 2018. MNRAS, in press (arXiv1805.07100)

Talon S, Charbonnel C. 2005. Astron. Astrophys. 440:981-994

Talon S, Charbonnel C. 2008. Astron. Astrophys. 482:597-605

Talon S, Kumar P, Zahn JP. 2002. Astrophys. J. Lett. 574:L175-L178

Talon S, Zahn JP, Maeder A, Meynet G. 1997. Astron. Astrophys. 322:209-217

Tassoul M. 1980. Astrophys. J. Suppl. Ser. 43:469-490

Tayar J, Pinsonneault MH. 2013. Astrophys. J. Lett. 775:L1

Tayar J, Pinsonneault MH. 2018. Astrophys. J., submitted (arXiv1806.10649)

Tayler RJ. 1975. Bull. of the American Astron. Soc. 7:252

Taylor GI. 1923. Philosophical Transactions of the Royal Society of London Series A 223:289-343

Thorpe SA. 2018. Journal of Fluid Mechanics 836:72-116

Tkachenko A, Degroote P, Aerts C, Pavlovski K, Southworth J, et al. 2014. MNRAS 438:3093-3110

Torres G, Andersen J, Giménez A. 2010. Astron. Astrophys. Rev. 18:67-126

Townsend AA. 1966. Journal of Fluid Mechanics 24:307-319

Townsend RHD. 2003a. MNRAS 343:125-136

Townsend RHD. 2003b. MNRAS 340:1020-1030

Townsend RHD, Goldstein J, Zweibel EG. 2018. MNRAS 475:879-893

Triana SA, Corsaro E, De Ridder J, Bonanno A, Pérez Hernández F, García RA. 2017. Astron. Astrophys. 602:A62

Triana SA, Moravveji E, Pápics PI, Aerts C, Kawaler SD, Christensen-Dalsgaard J. 2015. Astrophys. J. 810:16

Turck-Chièze S, Palacios A, Marques JP, Nghiem PAP. 2010. Astrophys. J. 715:1539-1555

Unno W, Osaki Y, Ando H, Saio H, Shibahashi H. 1989. Nonradial oscillations of stars, Tokyo: University of Tokyo Press, 1989, 2nd ed.

Van Reeth T, Mombarg JSG, Mathis S, Tkachenko A, Fuller J, et al. 2018. Astron. Astrophys., in press (arXiv1806.03586)

Van Reeth T, Tkachenko A, Aerts C. 2016. Astron. Astrophys. 593:A120 
Van Reeth T, Tkachenko A, Aerts C, Pápics PI, Triana SA, et al. 2015. Astrophys. J. Suppl. 218:27 van Saders JL, Ceillier T, Metcalfe TS, Silva Aguirre V, Pinsonneault MH, et al. 2016. Nat. 529:181184

Viallet M, Meakin C, Arnett D, Mocák M. 2013. Astrophys. J. 769:1

Viallet M, Meakin C, Prat V, Arnett D. 2015. Astron. Astrophys. 580:A61

Viani LS, Basu S, Joel Ong J. M, Bonaca A, Chaplin WJ. 2018. Astrophys. J. 858:28

von Zeipel H. 1924. MNRAS 84:665-683

Wade GA, Neiner C, Alecian E, Grunhut JH, Petit V, et al. 2016. MNRAS 456:2-22

White TR, Huber D, Mann AW, Casagrande L, Grunblatt SK, et al. 2018. MNRAS 477:4403-4413

Zahn J, Talon S, Matias J. 1997. Astron. Astrophys. 322:320-328

Zahn JP. 1975. Astron. Astrophys. 41:329-344

Zahn JP. 1983. Instability and Mixing Processes in Upper Main Sequence Stars. In Saas-Fee Advanced Course 13: Astrophysical Processes in Upper Main Sequence Stars, eds. AN Cox, S Vauclair, JP Zahn

Zahn JP. 1991. Astron. Astrophys. 252:179-188

Zahn JP. 1992. Astron. Astrophys. 265:115-132

Zahn JP. 2013. Rotation induced mixing in stellar interiors. In EAS Publications Series, eds. G Alecian, Y Lebreton, O Richard, G Vauclair, vol. 63 of EAS Publications Series

Zahn JP, Brun AS, Mathis S. 2007. Astron. Astrophys. 474:145-154

Zinn JC, Pinsonneault MH, Huber D, Stello D. 2018. Astrophys. J., submitted (arXiv1805.02650)

Zorec J, Royer F. 2012. Astron. Astrophys. 537:A120 


\section{Online Material}

In Figure 7, we show $v_{\theta}$ and the temperature fluctuations corresponding with a few of the quadrupole wave components of low order occurring in the generated IGW spectrum for a $3 \mathrm{M}_{\odot}$ main sequence star computed by Edelmann et al. (submitted). From Figures 6 and 7 , we deduce typical velocities of tens of $\mathrm{cms}^{-1}$ per individual wave component, and accompanying temperature fluctuations of a few K. These type of data are currently being used so simulate observables, such as photometric light curves for the Kepler and TESS passbands and synthetic high-resolution line-profile variations for comparison with optical spectroscopy of OB-type stars. In this way, we will evaluate whether or not the generated IGW spectra can explain the recent detections of low-frequency power excess in such data of OB-type stars. Earlier, Aerts et al. (2009) already showed that the collective effect of an ensemble of low-amplitude coherent $\mathrm{g}$ modes of degree $l=1, \ldots, 10$ offers a physical explanation for the observed line-profile broadening in some high-mass stars, generally called macroturbulence (e.g. Simón-Díaz \& Herrero 2014). This is evidence that $\mathrm{g}$ modes are not only appealing as an explanation for the recent asteroseismic measurements of $\Omega_{\text {core }}$, but can also explain features detected in time-series spectroscopy of high-mass stars that remain unexplained otherwise. The ongoing simulation study on the basis of the IGW generated by Edelmann et al. (submitted) offers a similar test for IGW instead of coherent $\mathrm{g}$ modes, from confrontation of the simulated diagnostics with space photometric light curves as well as spectroscopic measurements of OB-type stars.
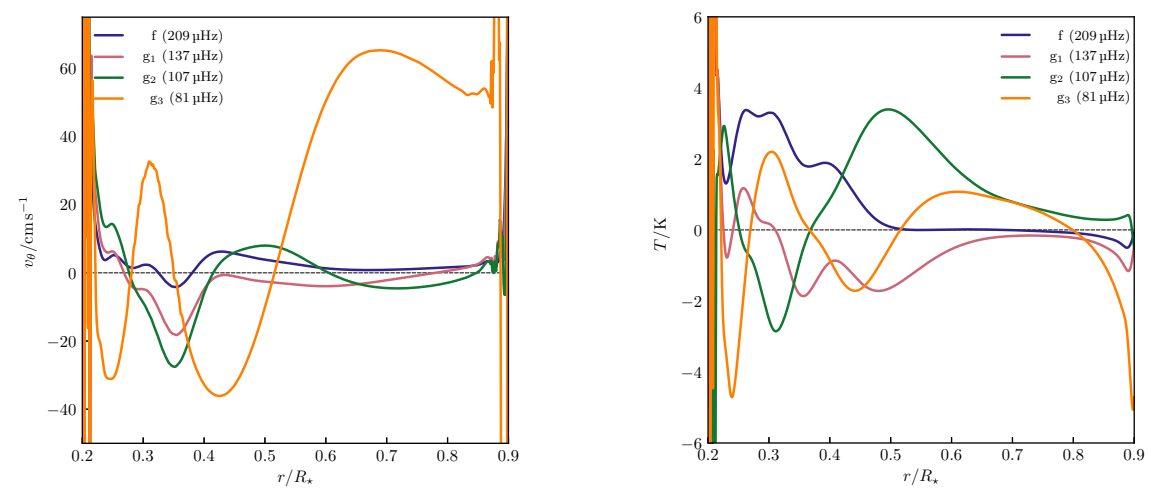

Figure 7

The $\theta$ component of the horizontal velocity (left panel) and the temperature fluctuation (right panel) for four of the coherent quadrupole mode components present in the IGW spectrum generated by the core convection, with their indicated frequencies. The radial velocity component of these four modes is shown in Figure 6 in the main text. Figure produced from data in Edelmann et al. (submitted). 